\title{
Optimal Pricing Strategy for Operators in Cognitive Femtocell Networks
}

\author{
Chunxiao Jiang, Member, IEEE, Yan Chen, Member, IEEE, K. J. Ray Liu, Fellow, IEEE, and \\ Yong Ren, Member, IEEE
}

\begin{abstract}
Cognitive femtocell has been envisioned as a promising technology for covering indoor environment and assisting heavy-loaded macrocell network. Although lots of technical issues of cognitive femtocell network have been studied, e.g., spectrum sharing, interference mitigation, etc., the economic issues that are very important for practical femtocell deployment have not been well investigated in the literatures. In this paper, we focus on the pricing issues in the cognitive femtocell network and propose a two-tier pricing game theoretic framework with two models: static and dynamic pricing models. In the static pricing model, we derive the closed-form expressions for pricing and demand functions, as well as the Nash equilibrium pricing strategies for both macrocell and femtocell operators. In the dynamic pricing model, we first model the cognitive users' network access behavior as a two-dimensional Markov decision process and propose a modified value iteration algorithm to find the best strategy profiles for cognitive users. Based on the analysis of users' behavior, we further design an iterative gradient descent algorithm to find the Nash equilibrium pricing strategies for both macrocell and femtocell operators. Simulation results verify our theoretic analysis and show that the proposed algorithm in the dynamic pricing model can quickly converge to the Nash equilibrium prices.
\end{abstract}

Index Terms - Cognitive femtocell, two-tier pricing, Nash equilibrium price, Markov decision process.

\section{INTRODUCTION}

$\mathbf{R}$ ECENTLY, femtocell technology is proposed to combat the poor signal reception problem for indoor environment and aims to provide high-speed, low-power communication solutions. Since it is predicted that in the near future, about $60 \%$ of voice traffic will originate from indoor environments with femtocell, femtocell networks have to use the same spectrums with macrocells to avoid additional hardware support [1]. Under such circumstance, the application of cognitive radio technology in the femtocell network becomes a promising solution [2]. The involvement of cognitive radio in femtocell not only can improve the efficiency of spectrum management, but

Manuscript received September 12, 2013; revised December 17, 2013 and April 4, 2014; accepted May 26, 2014. Date of publication June 3, 2014; date of current version September 8, 2014. This work was done at University of Maryland. This work was supported in part by the NSFC China under Projects 61371079, 61273214, and 61271267, by the National Basic Research Program of China (973 Program) under Grant 2013CB329105, and by the China Postdoctoral Science Foundation funded project. The associate editor coordinating the review of this paper and approving it for publication was S. Cui.

C. Jiang and Y. Ren are with the Department of Electronic Engineering, Tsinghua University, Beijing 100084, P. R. China (e-mail: chx.jiang@ gmail.com; reny@tsinghua.edu.cn).

Y. Chen and K. J. R. Liu are with the Department of Electrical and Computer Engineering, University of Maryland, College Park, MD 20742 USA (e-mail: yan@umd.edu; kjrliu@umd.edu).

Color versions of one or more of the figures in this paper are available online at http://ieeexplore.iee.org.

Digital Object Identifier 10.1109/TWC.2014.2327970 also enhance the flexibility and reconfigurability of femtocell's access point (AP) deployment [3], [4].

The concept of "cognitive femtocell" has received more and more attentions from researchers around the world. In [4]-[6], downlink spectrum sharing problems between macrocells and femtocells were studied, including joint channel allocation and power control method in [5], decentralized OFDMA based cognitive femtocell network in [6] and underlay spectrum sharing schemes design and analysis in [7]. To mitigate the interference in cognitive femtocell network, Chang proposed a multiple-access CDMA based approach in [8] and Wang et al. proposed a stochastic dual control approach in [9]. Game-theoretic resource allocation in cognitive femtocell network are studied in [10] with correlated equilibrium analysis and in [11] with Stackelberg game model. In [12], two cooperation models, called as "Cooperative Relay Model" and "Interference Model", were proposed for femtocell and macrocell users in cognitive femtocell networks. Moreover, $\mathrm{Hu}$ et al. discussed the scenario of scalable video streaming over cognitive femtocell network in [13].

Those prior works on cognitive femtocells mainly focused on various technical issues, such as spectrum sharing, interference mitigation, resource allocation, etc. Little effort has been made on the economic issue in cognitive femtocell networks, which is also an important topic when it comes to practical deployment of cognitive femtocells. In this paper, we will study the pricing game between macrocell operator and femtocell operator in cognitive femtocell networks. In the literatures, the pricing related issues were discussed in [13] and [14], where Yun et al. designed two pricing schemes (flat and partial volume pricing) under the case of monopoly market in [14] and Kang et al. analyzed two pricing models (uniform and non-uniform pricing) under two scenarios (sparsely and densely deployed scenarios) in [15]. From the perspective of operators, Ren et al. discussed whether the operator should enter the femtocell market in [16], while Duan et al. studied whether the operator should provide users with only the macrocell service, only the femtocell service, or both services in [17].

All the existing literatures assume that both femtocell and macrocell are operated by the same service provider. However, the femtocell operator and macrocell operator can be different in the practical scenarios. For example, in China, China Telecom, the third largest wireless service provider is planning to lease TD-LTE spectrums from China Mobile, the largest wireless service provider, and build femtocell networks [18]. In such a case, each operator only tries to maximize its own utility and the competition between the macrocell and femtocell operators would appear, which is the main difference of this work from the traditional heterogeneous networks. In this paper, we consider such a scenario and study the pricing 
issue among indoor users, femtocell operator and macrocell operator. Although the spectrum leasing related issues were analyzed in [18] and [19], including the macrocell-femtocell cooperation game in [19] and hybrid access based spectrum leasing framework in [20], the pricing issue was not discussed in these works. Note that the spectrum leasing game is also investigated in cognitive radio networks, where the primary users lease licensed spectrums to the secondary users with some [21]-[23]. In our model, the cognitive users can choose to access either femtocell or macrocell, which is the main difference from the existing spectrum leasing game in cognitive radio networks where the secondary users can only access the secondary networks. Moreover, there is also competition between the femtocell and macrocell operators in our model, leading to a two-tier pricing game model which is totally different from the spectrum leasing game in cognitive radio networks.

In this paper, we study the pricing game between macrocell and femtocell operators, where femtocell operators lease spectrums from the macrocell operator to provide high-speed communications for indoor users. Given the spectrum leasing price, macrocell and femtocell operators set the network access prices independently and non-cooperatively. The cognitive users can access either macrocell network or femtocell network to achieve the best utility. We formulate this pricing problem as a two-tier pricing model and derive the Nash equilibrium prices under two models: static pricing model and dynamic pricing model, where "dynamic" means that the network access price is changing with the number of users in the network. The main contributions of this paper are summarized as follows.

1) We propose a two-tier pricing game framework to study the pricing strategies of macrocell and femtocell operators. The two-tier pricing game is analyzed under two models: static pricing model and dynamic pricing model. Based on the analysis of cognitive users' network access behaviors, we derive the utilities of both operators and the Nash equilibrium pricing strategies.

2) In static pricing model, we define the cognitive users' utility functions of accessing macrocell or femtocell network, as well as macrocell and femtocell operators' utility functions. According to the utility functions, we further derive the closed-form expressions for pricing and demand functions of both operators. Through solving the joint pricing equations, the closed-form expressions for Nash equilibrium pricing strategies of both operators are derived.

3) In dynamic pricing model, we analyze cognitive users' network access behavior by considering their long-term expected utilities and the interactions among them due to negative network externality. We formulate users' network access behavior as a 2-D Markov decision process, and propose a modified value iteration algorithm to find the corresponding best strategy profile. We further theoretically prove that there exists a threshold structure in the best strategy profile. Moreover, based on the analysis of cognitive users' behaviors, we analyze the utilities of macrocell and femtocell operators, and design an iterative gradient descent algorithm to find the Nash equilibrium pricing strategies for both operators.

The rest of this paper is organized as follows. First, our system model is described in Section II. Then, we analyze the

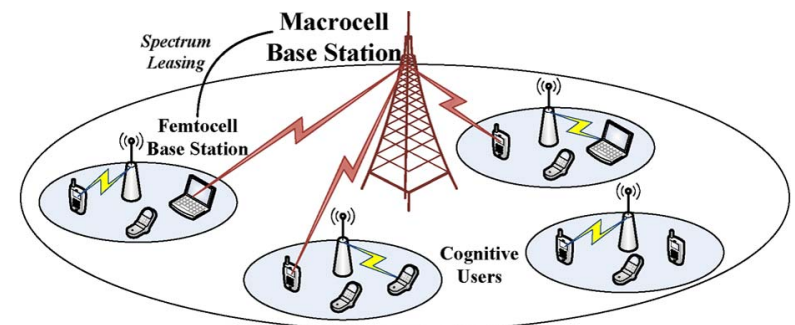

Fig. 1. System model of the cognitive femtocell network.

Nash equilibrium pricing strategies of macrocell and femtocell operators in terms of static and dynamic pricing model in Sections III and IV, respectively. Finally, simulation results are shown in Section V and conclusions are drawn in Section VI.

\section{SySTEM MOdEL}

\section{A. Network Entity}

The system diagram is shown in Fig. 1. We consider one macrocell base station located at the center of one region, which is operated on licensed spectrums and considered as licensed users in the system. Within the coverage area of the macrocell base station, there are multiple femtocell networks operating on spectrums which are leased from macrocell network. Note that there is no interference between macrocell and femtocell network since they are operated on different spectrums, i.e., the interweave heterogeneous networks architecture [24], [25]. The spectrum leasing price, denoted by $w$, is set by the macrocell operator. The macrocell network and femtocell network offer different spectrum access prices to the mobile users, denoted by $p_{m}$ and $p_{f}$, respectively. For users within the coverage area of one femtocell base station, they can cognitively access either femtocell network or macrocell network, i.e., the "open access" model [1]. As shown in Fig. 1, since all femtocell networks are operated by the same operator and thus relatively homogeneous, we only focus on one femtocell network and study the cognitive users' behaviors within it, based on which we further analyze its pricing strategies. Note that although the cognitive radio model in our system is based on "spectrum leasing" scheme instead of "spectrum sensing" scheme, all the results in this paper can be easily extended to those of the latter scheme.

\section{B. Two-Tier Pricing Model}

According to the system diagram discussed above, we can abstract a two-tier pricing model as shown in Fig. 2. For cognitive users, on one hand, accessing femtocell network may obtain higher data rate but with higher payment due to additional spectrum leasing costs for the femtocell operator. On the other hand, accessing macrocell network can lead to lower payment but users may experience unsatisfied data rate due to unfavorable locations. Therefore, rational cognitive users make their decisions, i.e., accessing macrocell network or femtocell network, by comparing the corresponding utilities.

For network operators (macrocell and femtocell operators), on one hand, through adjusting the network access prices $p_{m}$ and $p_{f}$, they compete with each other for more users to maximize their own profits. On the other hand, the macrocell operator can also influence femtocell's pricing strategy through 


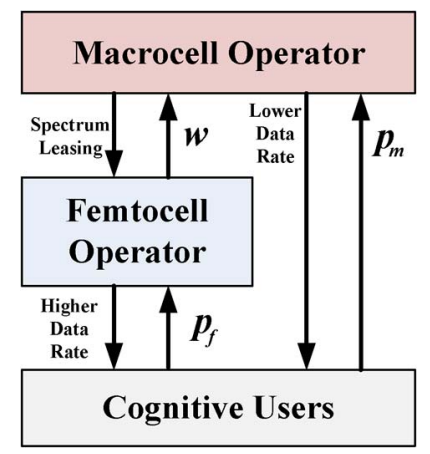

Fig. 2. Two-tie pricing model.

controlling the spectrum leasing price $w$. In such a case, we can formulate the two-tier pricing game model as a non-cooperative game with complete information as follows:

- Players: The macrocell operator and femtocell operator.

- Strategies: The two players' strategies are network access prices set by two network operators: $p_{m}$ and $p_{f}$, respectively. That is, the strategy profile is $\left(p_{m}, p_{f}\right)$. Note that $p_{f}$ should be no less than the spectrum leasing price, i.e., $p_{f}>w$. (This is because if $p_{f} \leq w$, the femtocell operator's profit will be negative or 0 .)

- Utilities: The utilities of macrocell and femtocell operators, denoted by $V_{m}\left(p_{m}, p_{f}\right)$ and $V_{f}\left(p_{m}, p_{f}\right)$, are their own overall profits, respectively.

Based on the game formulation above, we can define the Nash Equilibrium (NE) of this two-tie pricing game as follows.

Definition 1-Nash Equilibrium Price (NE Price): A price strategy profile $\left(p_{m}^{N}, p_{f}^{N}\right)$ is a NE pricing strategy profile of the two-tie pricing game, if and only if, no unilateral deviation in pricing strategy by any single operator is profitable for that operator, that is

$$
\begin{array}{cc}
\forall p_{m}, & V_{m}\left(p_{m}^{N}, p_{f}^{N}\right) \geq V_{m}\left(p_{m}, p_{f}^{N}\right), \\
\forall p_{f}>w, & V_{f}\left(p_{m}^{N}, p_{f}^{N}\right) \geq V_{f}\left(p_{m}^{N}, p_{f}\right) .
\end{array}
$$

In the following section, we will study the NE price under two models: static pricing model and dynamic pricing model. In static pricing model, the prices $\left(p_{m}, p_{f}\right)$ are independent of the network state, i.e., the number of users in each network, and users' utilities are independent of each other, which means that the negative externality is not considered. With the static pricing model, we can derive closed-form expression for $\mathrm{NE}$ price to give insights of the relationship between $w, p_{m}$ and $p_{f}$. In dynamic pricing model, the prices $\left(p_{m}, p_{f}\right)$ depend on the number of users in each network, and the more users access one network, the less utility each user can obtain, which means that the negative externality is considered.

\section{Static Pricing Model Without Negative EXTERNALITY}

In this section, we study the static pricing model, where the network access prices for macrocell network and femtocell network, $p_{m}$ and $p_{f}$, are independent of the number of users sharing the same network. Moreover, the negative externality is not considered in this static model, which means users' throughput are independent of each other [26], [27]. According to the Hotelling model in economics [28], which is usually

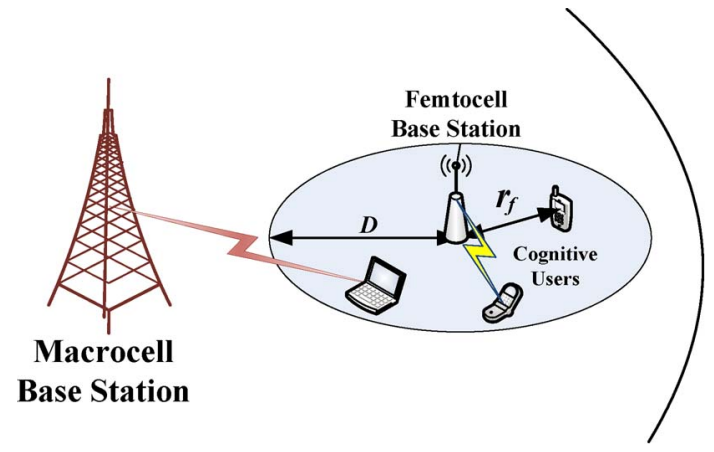

Fig. 3. Static pricing model.

used to model the distribution of customers within one area, we assume that cognitive users are linearly uniformly located within the coverage of each femtocell base station. As shown in Fig. 3, suppose the coverage radius of each femtocell is $D$ and the number of all cognitive users within the femtocell is $N_{D}$. Let $r_{f}$ represent the distance between a cognitive user and the corresponding femtocell base station. With the Hotelling model, the number of users located within distance $r_{f} \leq d$, $N_{d}$, satisfies that $\left(N_{d} / N_{D}\right)=(d / D)$. Note that our analysis in this paper is not limited to be the Hotelling model, and other user distribution model can be analyzed in a similar way. In the following derivation, we normalize the coverage radius of each femtocell, $D$, to be 1 , i.e., the distance between a user and the corresponding femtocell base station, $r_{f}$, satisfies $0<r_{f} \leq 1$.

\section{A. Utility Functions}

1) Utility Functions of Cognitive Users: When confronted with macrocell network and femtocell network simultaneously, a rational cognitive user would estimate and compare the utilities from accessing both networks and make a selection that can provide better utility. From a rational cognitive user's perspective, his/her motivation is always to acquire as much data rate as possible with a low cost, where the cost mainly refers to the network access fees, as well as the interference from neighboring cells. In such a case, given network access prices $p_{m}$ and $p_{f}$, we can define the utility functions of users located at $r_{f}$ as follows:

$$
\begin{aligned}
U_{m} & =R_{m}-\theta p_{m}-I_{m}, \\
U_{f} & =R_{f}-\theta p_{f}-r_{f} I_{f},
\end{aligned}
$$

where $U_{m}$ and $U_{f}$ are utilities of choosing macrocell network and femtocell network, respectively; $R_{m}$ and $R_{f}$ are throughput each cognitive user can obtain by accessing macrocell network and femtocell network, respectively; $I_{m}$ is the interference from neighboring macrocells, which is considered as a constant within the coverage of one femtocell since the coverage radius of one femtocell is usually 20 to 50 meters, while the coverage radius of one macrocell is typically around 1 kilometer [29]; $r_{f} I_{f}$ is the interference from neighboring femtocells, which is considered proportionally to the distance between the user and the femtocell base station, since the further from the femtocell base station, the more interference will be caused and the interference reaches limit on the boundary of two adjacent femtocells; and $\theta$ is a constant coefficient that transfers the price cost into throughput loss. Note that the interference terms 
$I_{m}$ and $I_{f}$ in the utility functions represents the throughput degradation due to the inter-cell interference, e.g., $I_{m}$ can be calculated as follows:

$$
I_{m}=\log \left(1+\frac{P_{s}}{P_{n}}\right)-\log \left(1+\frac{P_{s}}{P_{n}+P_{i}}\right),
$$

where $P_{s}$ denotes the macrocell signal power, $P_{n}$ denotes the noise power and $P_{i}$ denotes the inter-cell interference power. Such a utility definition is also helpful to derive the closedform expression of the NE price, which can give more insightful results about the static pricing model. The cognitive users consider to access the network only if the utility is larger than 0 . If both $U_{m}$ and $U_{f}$ are positive, the user will choose the one with larger utility. From the utility function definitions in (3) and (4), we can see that the price $p_{f}$ should satisfy $w<p_{f}<$ $R_{f} / \theta$, otherwise, no user will choose femtocell network and the two-tier pricing model will not exist.

2) Utility Functions of Macrocell and Femtocell Operators: Suppose the numbers of cognitive users choosing the macrocell network and the femtocell network are $Q_{m}$ and $Q_{f}$, respectively. Note that the total number of users within the coverage of one femtocell is normalized as 1 , which means that $Q_{m}+$ $Q_{f} \leq 1$. As shown in Fig. 2, the femtocell operator's utility is only determined by femtocell users' access. On the other hand, for the macrocell operator, both channels of users' access can contribute to its utility. In such a case, the utility functions of both operators, $V_{m}$ and $V_{f}$, can be defined as follows:

$$
\begin{aligned}
V_{m} & =w Q_{f}+p_{m} Q_{m}, \\
V_{f} & =\left(p_{f}-w\right) Q_{f} .
\end{aligned}
$$

Recall that $w$ is the spectrum leasing price. With the utility function definitions in (3)(7), we will analyze the NE price as defined in Section II-B in the following.

\section{B. Best Response of Femtocell Network}

In this subsection, we first derive the best response of femtocell network, i.e., optimal pricing strategy $p_{f}^{*}$ when given the network access price of macrocell network, $p_{m}$. According to users' utility of accessing macrocell network, $U_{m}$, there are two cases:

- Case 1: $p_{m} \geq\left(R_{m}-I_{m}\right) / \theta$

In this case, according to (3), $U_{m} \leq 0$, which means that all users will not choose macrocell network, i.e., $Q_{m}=0$. On the other hand, one cognitive user will access femtocell network if and only if his/her utility $U_{f}$ is larger 0 . In such a case, according to (4), we have $r_{f} \leq\left(R_{f}-\theta p_{f}\right) / I_{f}$ which means that only users located at a distance less than $\left(R_{f}-\theta p_{f}\right) / I_{f}$ from the femtocell base station will access femtocell network. Since the total number of users within one femtocell has been normalized as 1 , we can derive the demand function of femtocell network, $Q_{f}$, as follows:

$$
Q_{f}= \begin{cases}1, & \text { if } w<p_{f} \leq\left(R_{f}-I_{f}\right) / \theta, \\ \left(R_{f}-\theta p_{f}\right) / I_{f}, & \text { if }\left(R_{f}-I_{f}\right) / \theta<p_{f}<R_{f} / \theta .\end{cases}
$$

From (8), we can see that the demand function $Q_{f}$ is a nonincreasing function in terms of price $p_{f}$ and all users will choose to access femtocell network when $p_{f}$ is lower than the threshold $\left(R_{f}-I_{f}\right) / \theta$. To obtain the optimal pricing strategy, $p_{f}^{*}$, which maximizes the utility of operators, we can solve the equation arg $\max _{p_{f} \in\left(w, R_{f} / \theta\right)} V_{f}=\left(p_{f}-w\right) Q_{f}$ as

$$
p_{f}^{*}= \begin{cases}\left(R_{f}-I_{f}\right) / \theta, & \text { if } w \leq\left(R_{f}-2 I_{f}\right) / \theta, \\ \left(\theta w+R_{f}\right) / 2 \theta, & \text { if }\left(R_{f}-2 I_{f}\right) / \theta<w<R_{f} / \theta .\end{cases}
$$

And the corresponding optimal demand $Q_{f}^{*}$ is

$$
Q_{f}^{*}= \begin{cases}1, & \text { if } w \leq\left(R_{f}-2 I_{f}\right) / \theta, \\ \left(R_{f}-\theta w\right) / 2 I_{f}, & \text { if }\left(R_{f}-2 I_{f}\right) / \theta<w<R_{f} / \theta .\end{cases}
$$

- Case 2: $p_{m} \leq\left(R_{m}-I_{m}\right) / \theta$

In this case, according to (3), we have $U_{m} \geq 0$, and thus the influence of $p_{m}$ on $p_{f}$ should be taken into account. Since users will access the network which can provide higher utility, through comparing $U_{m}$ and $U_{f}$ in (3) and (4), we have (11), shown at the bottom of the page. Similarly, the optimal pricing strategy $p_{f}^{*}$ is given in (12), shown at the bottom of the page. From (9) and (12), we can see given the pricing strategy of macrocell network $p_{m}$, the optimal pricing strategy for femtocell network $p_{f}^{*}$ is a non-decreasing function in terms of spectrum leasing price $w$.

\section{Best Response of Macrocell Networks}

In this subsection, we derive the best response of macrocell network, i.e., optimal pricing strategy $p_{m}^{*}$ when given the network access price of femtocell network, $p_{f}$. According to the relationship between $U_{m}$ and $U_{f}$, we consider three intervals of $p_{m}$ as follows.

Interval 1: $p_{m} \leq p_{f}+\left(R_{m}-R_{f}-I_{m}\right) / \theta$, within which $U_{f}$ is always less than $U_{m}$. In such a case, $Q_{f}=0, Q_{m}=1$, and $V_{m}=p_{m}$ which is an increasing function in terms of $p_{m}$.

Interval 2: $p_{f}+\left(R_{m}-R_{f}-I_{m}\right) / \theta<p_{m}<p_{f}+\left(R_{m}-\right.$ $\left.R_{f}+I_{f}-I_{m}\right) / \theta$. According to whether $U_{f}$ is larger than 0 , there are two cases.

- Case 1: $w<p_{f} \leq\left(R_{f}-I_{f}\right) / \theta$

In this case, $U_{f}$ is always larger than 0 , and $p_{m}<$ $\left(R_{m}-I_{m}\right) / \theta$, i.e., $U_{m}>0$. Therefore, through comparing $U_{f}$ and $U_{m}$, we have $Q_{f}=\left(R_{f}-R_{m}+I_{m}+\theta p_{m}-\theta p_{f}\right) / I_{f}$,

$$
Q_{f}= \begin{cases}1, & \text { if } w<p_{f} \leq\left(R_{f}-R_{m}+I_{m}-I_{f}+\theta p_{m}\right) / \theta, \\ \left(R_{f}-R_{m}+I_{m}+\theta p_{m}-\theta p_{f}\right) / I_{f}, & \text { if }\left(R_{f}-R_{m}+I_{m}-I_{f}+\theta p_{m}\right) / \theta<p_{f}<R_{f} / \theta\end{cases}
$$

$$
p_{f}^{*}= \begin{cases}\left(R_{f}-R_{m}+I_{m}-I_{f}+\theta p_{m}\right) / \theta, & \text { if } w \leq\left(R_{f}-R_{m}+I_{m}-2 I_{f}+\theta p_{m}\right) / \theta, \\ \left(R_{f}-R_{m}+I_{m}+\theta w+\theta p_{m}\right) / 2 \theta, & \text { if }\left(R_{f}-R_{m}+I_{m}-2 I_{f}+\theta p_{m}\right) / \theta<w<R_{f} / \theta\end{cases}
$$


$Q_{m}=1-Q_{f}$. In such a case, the utility of macrocell operator is

$$
V_{m}=w Q_{f}+p_{m}\left(1-Q_{f}\right)=\left(w-p_{m}\right) Q_{f}+p_{m} .
$$

Since $\left(\partial^{2} V_{m} / \partial p_{m}^{2}\right)=-2 \theta<0, V_{m}$ is a concave function in terms of $p_{m}$ in this case. The optimal $p_{m}^{*}$ can be calculated by solving $\left(\partial V_{m} / \partial p_{m}\right)=0$ as

$$
p_{m}^{*}=\left(R_{m}-R_{f}+I_{f}-I_{m}+\theta w+\theta p_{f}\right) / 2 \theta .
$$

- Case 2: $\left(R_{f}-I_{f}\right) / \theta<p_{f}<R_{f} / \theta$

In this case, if $p_{m}>\left(R_{m}-I_{m}\right) / \theta$, the utility of macrocell operator is independent of $p_{m}$ since $U_{m}<0$ and $V_{m}=w Q_{f}$. Therefore, we only consider when $p_{m} \leq\left(R_{m}-I_{m}\right) / \theta$, which is similar with Case 1 that $V_{m}$ is a concave function in terms of $p_{m}$ and the optimality achieves at the same point.

Interval 3: $p_{m} \geq p_{f}+\left(R_{m}-R_{f}+I_{f}-I_{m}\right) / \theta$, which means $U_{f}$ is always larger than $U_{m}$. In such a case, $Q_{m}=0$ and $V_{m}=w Q_{f}$ is independent of $p_{m}$.

To summarize, we can see that $V_{m}$ is continuous in Interval 1 and Interval 2, and $V_{m}$ is increasing in Interval 1 and concave in Interval 2. In Interval $3, V_{m}$ is independent of $p_{m}$ and is less than $V_{m}$ at the boundary point of Interval 2 . Therefore, the optimal $p_{m}^{*}$ should lie within Interval 2 or at the boundary of Interval 2 . In such a case, we can summarize the best response function of macrocell operator as follows:

1) When $w \leq\left(R_{m}-I_{m}-I_{f}\right) / \theta, p_{m}^{*}$ is given in (15), shown at the bottom of the page.

2) When $w>\left(R_{m}-I_{m}-I_{f}\right) / \theta, p_{m}^{*}$ is given in (16), shown at the bottom of the page.

Similarly, we can see given the pricing strategy of femtocell network $p_{f}$, the optimal pricing strategy for macrocell network $p_{m}^{*}$ is also a non-decreasing function in terms of spectrum leasing price $w$.

\section{NE Price}

In this subsection, we analyze the NE price for both macrocell and femtocell operators, $\left(p_{m}^{N}, p_{f}^{N}\right)$, based on the best response functions we derived in previous subsections. Since when $p_{m}>w>\left(R_{m}-I_{m}\right) / \theta$, the price of accessing macrocell network is so high that no cognitive user will access it, we only consider the case of $w \leq\left(R_{m}-I_{m}\right) / \theta$ in the following theorem, where the NE prices under different conditions are summarized.
Theorem 1: When the spectrum leasing price $w \leq\left(R_{m}-\right.$ $\left.I_{m}\right) / \theta$, the NE price $\left(p_{m}^{N}, p_{f}^{N}\right)$ is

1) when $I_{m}+R_{f}-R_{m}<2 I_{f}$ and $w<\left(R_{f}+2 R_{m}-\right.$ $\left.2 I_{f}-2 I_{m}\right) / 3 \theta$,

$$
\left\{\begin{array}{l}
p_{m}^{N}=w+\left(R_{m}-R_{f}+2 I_{f}-I_{m}\right) / 3 \theta \\
p_{f}^{N}=w+\left(R_{f}-R_{m}+I_{f}+I_{m}\right) / 3 \theta
\end{array}\right.
$$

2) when $I_{m}+R_{f}-R_{m}<2 I_{f}$ and $w \geq\left(R_{f}+2 R_{m}-\right.$ $\left.2 I_{f}-2 I_{m}\right) / 3 \theta$,

$$
\left\{\begin{array}{l}
p_{m}^{N}=\left(R_{m}-I_{m}\right) / \theta \\
p_{f}^{N}=\left(\theta w+R_{f}\right) / 2 \theta
\end{array}\right.
$$

3) when $I_{m}+R_{f}-R_{m} \geq 2 I_{f}$,

$$
\left\{\begin{array}{l}
p_{m}^{N}=w \\
p_{f}^{N}=w+\left(R_{f}-R_{m}+I_{m}-I_{f}\right) / \theta
\end{array}\right.
$$

The physical meaning of the NE price is a pricing point $\left(p_{m}, p_{f}\right)$ where each operator's utility is maximized given the other operator's pricing strategy. Therefore, the NE price can be found by calculating the intersection point of those two network operators' best response functions, i.e., (9), (12) and (15), (16). Due to page limit, we omit the derivation details here. Note that when there is no intersection, the boundary point is taken as NE, as shown in Case 3) of Theorem 1, since the boundary point $p_{m}=w$ already maximizes the utility of the macrocell operator. Moreover, since all the best response functions are linear as shown in (9), (12) and (15), (16), the intersection of two linear functions can only be unique, which guarantees the uniqueness of the NE price.

\section{E. Discussions}

In this subsection, we discuss some physical meanings behind Theorem 1. If we regard $R_{f}-R_{m}$ as the potential cost of accessing macrocell network instead of femtocell network, then $I_{m}+R_{f}-R_{m}$ in Theorem 1 can be considered as the overall cost of accessing macrocell network. Based on this perspective, we can interpret different NE price under different conditions as different states between the operators of both networks. In the following, we will discuss four different states between two operators: "independent state", "competition state", "cooperation state" and "threatening state", where each of them is corresponding to one specific NE price.

\section{- State 1: Independent State}

When the macrocell operator increases the spectrum leasing price $w$ as to $w>\left(R_{m}-I_{m}\right) / \theta$, no user will access macrocell

$$
p_{m}^{*}= \begin{cases}w, & \text { if } p_{f} \leq\left(R_{f}-R_{m}+I_{m}-I_{f}+\theta w\right) / \theta \\ \left(R_{m}-R_{f}+I_{f}-I_{m}+\theta w+\theta p_{f}\right) / 2 \theta, & \text { if }\left(R_{f}-R_{m}+I_{m}-I_{f}+\theta w\right) / \theta<p_{f}<\left(R_{f}-R_{m}+I_{m}+I_{f}+\theta w\right) / \theta \\ p_{f}+\left(R_{m}-R_{f}-I_{m}\right) / \theta, & \text { if }\left(R_{f}-R_{m}+I_{m}+I_{f}+\theta w\right) / \theta \leq p_{f} \leq R_{f} / \theta\end{cases}
$$

$$
p_{m}^{*}= \begin{cases}w, & \text { if } p_{f} \leq\left(R_{f}-R_{m}+I_{m}-I_{f}+\theta w\right) / \theta \\ \left(R_{m}-R_{f}+I_{f}-I_{m}+\theta w+\theta p_{f}\right) / 2 \theta, & \text { if }\left(R_{f}-R_{m}+I_{m}-I_{f}+\theta w\right) / \theta<p_{f}<\left(R_{f}+R_{m}-I_{m}-I_{f}-\theta w\right) / \theta \\ \left(R_{m}-I_{m}\right) / \theta, & \text { if }\left(R_{f}+R_{m}-I_{m}-I_{f}-\theta w\right) / \theta \leq p_{f} \leq R_{f} / \theta\end{cases}
$$


network since $p_{m}>w>\left(R_{m}-I_{m}\right) / \theta$ and $U_{m}<0$. In such a case, from cognitive users' perspectives, macrocell network is kind of invisible and femtocell network seems to be monopoly. Meanwhile, the utilities of both network operators are independent with each other, as well as their pricing strategies, due to which we define this state as "independent state". Although there is no user accessing macrocell network, the macrocell operator can already obtain sufficient utilities from leasing spectrums to the femtocell operator with high spectrum leasing price. For femtocell network, in order to ensure positive utility, the price it offers to users $p_{f}$ should be larger than $w$, i.e., $\left(p_{f}>w\right)$, due to which many users will not access it neither. In other words, such a strategy leads to low utilities for both operators. Therefore, this "independent state" is not a desirable NE price for both operators.

\section{- State 2: Competition State}

When the overall cost of accessing macrocell network $I_{m}+$ $R_{f}-R_{m}$ is relatively low and the spectrum leasing price $w$ is also relatively low, the competition between macrocell operator and femtocell operator appears, i.e., the system is in "competition state" and the corresponding NE is shown in the first case of Theorem 1. It may be easier to see the competition by comparing the pricing strategy and demand of femtocell network between this state and "independent state". Let us denote the optimal pricing strategy and demand of femtocell operator in "independent state" as $p_{f}^{I}$ and $Q_{f}^{I}$, which are shown in (9) and (10), respectively. Then, we can see that the femtocell operator's pricing strategy in this state $p_{f}^{N}<p_{f}^{I}$ by comparing (17) and (9), and $Q_{f}^{C}=\left[w+\left(R_{f}-R_{m}+I_{f}+\right.\right.$ $\left.\left.I_{m}\right) / 3 \theta\right] / I_{f}<Q_{f}^{I}$. Such phenomenons reveal that the femtocell operator is no longer monopoly and it has to reduce its price to compete for more users.

\section{- State 3: Cooperation State}

When the overall cost of accessing macrocell network $I_{m}+$ $R_{f}-R_{m}$ is relatively low but the spectrum leasing price $w$ becomes higher, the cooperation between macrocell operator and femtocell operator appears, which leads to the "cooperation state" with the corresponding NE shown in the second case of Theorem 1. In this state, the optimal demand of femtocell network $Q_{f}^{N}$ is equal to $Q_{f}^{I}$, the demand of femtocell network in "independent state", which is the femtocell's monopoly state; while the demand of macrocell network is $Q_{m}^{N}=1-Q_{f}^{N}$. Such a phenomenon shows that users who are relatively near with the femtocell base station choose to access femtocell network, and the remaining users choose to access macrocell network. In such a case, both network operators cooperate to attract all users and the social welfare of this state is the highest compared with other states. Therefore, this "cooperation state" is a favorable NE price for both operators.

\section{- State 4: Threatening State}

When the overall cost of accessing macrocell network $I_{m}+$ $R_{f}-R_{m}$ is relatively high, no user accesses macrocell network, i.e., $Q_{m}^{N}=0$ and $Q_{f}^{N}=1$. One possible scenario is that the femtocell base station is located near the boundary of two macrocells, due to which users will suffer from sever interference from neighboring macrocell if accessing macrocell network. Compared with the "independent state", the optimal pricing strategy of femtocell network in this state satisfies

$$
p_{f}^{N}=w+\left(R_{f}-R_{m}+I_{m}-I_{f}\right) / \theta<\left(R_{f}-I_{f}\right) / \theta=p_{f}^{I}
$$

which means that although no user chooses to access macrocell network, the pricing of macrocell operator can threaten that of femtocell operator. Note that this "threatening state" is also not a desirable NE price for both operators.

\section{Dynamic Pricing Model With Negative EXTERNALITY}

In this section, we study the dynamic pricing model, where the negative externality is considered. In this model, the network access prices $\left(p_{m}, p_{f}\right)$ depends on the number of users in each network, and each user's utility depends on others' since the more users access one network, the less utility each user can obtain, i.e., negative network externality [26], [27]. Let us define the network state as $S=\left(i_{m}, i_{f}\right)$, where $i_{m}$ means the number of users in macrocell network and $i_{f}$ means that in femtocell network. Note that each network can only serve limited number of users simultaneously, thus we have $i_{m} \leq$ $N_{m}$ and $i_{m} \leq N_{f}$, where $N_{m}$ and $N_{f}$ are maximum numbers of users each network can support at one time, respectively. Here, we consider three kinds of users:

1) users who always access the macrocell network;

2) users who always access the femtocell network;

3) cognitive users who can access either the macrocell or femtocell network according to the utilities.

We assume that these three kinds of users arrive by Poisson process with arrival rate: $\lambda_{m}, \lambda_{f}$, and $\lambda$, respectively. After a user accesses one network, he/she cannot switch to the other network within a period of time exponentially distributed with mean $\mu^{-1}$, independent of which network the user accesses. We assume that there is a log-file in the server of each network, which records each user's access time and leaving time. Through querying this log-file, the new coming cognitive user can obtain current network state $S$, i.e., the number of users in each network, as well as the estimation of network parameters $\lambda_{m}, \lambda_{f}, \lambda$, and $\mu$.

The immediate utility functions of a cognitive user can be defined as follows:

$$
\begin{aligned}
U_{m}\left(i_{m}\right) & =R_{m}\left(i_{m}\right)-\theta p_{m}\left(i_{m}\right)-I_{m}, \\
U_{f}\left(i_{f}\right) & =R_{f}\left(i_{f}\right)-\theta p_{f}\left(i_{f}\right)-I_{f},
\end{aligned}
$$

where $R_{m}\left(i_{f}\right)=B_{m} \log \left(1+\left(\mathrm{SNR}_{m} /\left(i_{m}-1\right) \mathrm{INR}_{m}+1\right)\right)$ and $R_{f}\left(i_{f}\right)=B_{f} \log \left(1+\left(\mathrm{SNR}_{f} /\left(i_{f}-1\right) \mathrm{INR}_{f}+1\right)\right), B_{m}$ and $B_{f}$ are bandwidths; $\mathrm{SNR}_{m}$ and $\mathrm{INR}_{m}$ are Signal-NoiseRatio and Interference-Noise-Ratio from each of other users in the macrocell network, respectively, and $\mathrm{SNR}_{f}$ and $\mathrm{INR}_{f}$ are those in the femtocell network accordingly; $p_{m}\left(i_{m}\right)$ and $p_{f}\left(i_{f}\right)$ are pricing strategies of both operators, which are changing with the network state; $I_{m}$ and $I_{f}$ are interference caused by neighboring macrocells and femtocells, respectively, which are independent of the network state. Note that the interference from neighboring femtocells are considered as the worst case 
here. In this model, we consider the prices $p_{m}$ and $p_{f}$ satisfying that

$$
\begin{aligned}
& \forall i_{m} \leq N_{m}, \quad p_{m}\left(i_{m}\right)<R_{m}\left(N_{m}\right)-I_{m}, \\
& \forall i_{f} \leq N_{f}, \quad p_{f}\left(i_{f}\right)<R_{f}\left(N_{f}\right)-I_{f},
\end{aligned}
$$

which means that $U_{m}>0$ and $U_{f}>0$. Moreover, we assume that both immediate utility functions are decreasing functions in terms of network state, i.e., $\left(\partial U_{m}\left(i_{m}\right) / \partial i_{m}\right)<0$ and $\left(\partial U_{f}\left(i_{f}\right) / \partial i_{f}\right)<0$. This assumption means that the more users sharing one network, the less utility each user can obtain, which is consistent with practical scenarios. In the following, we will first analyze the cognitive users' (the third kind of users) network access behaviors, and then study the NE price for both operators.

\section{A. Cognitive Users' Network Access Behavior}

As discussed above, there are three classes of user arrival streams. Since the first two classes of users' network behaviors are fixed, i.e., regularly accessing macrocell or femtocell network, we only focus on analyzing the third class of users, i.e., cognitive users' network access behavior. For rational cognitive users, they will access a network that can provide higher utility. To achieve this goal, one user not only needs to consider the immediate utility defined in (21) and (22), but also takes into account the subsequent users' network access since the more subsequent users access the same network, the less utility that can be obtained in the future. In our model, users arrive by the Poisson process and access the network sequentially. When confronted with the network selection, a cognitive user only has the knowledge about current network state information, i.e., $\left(i_{m}, i_{f}\right)$. In order to take into account users' utility in the future, we use Bellman equation to formulate a user's utility and use Markov decision process (MDP) model to analyze the rational users' behaviors. In traditional MDP problem, a player can adjust his/her decision when the system state changes. However, in our system, once accessing one network, a user cannot adjust his/her decision even if the network state has already changed. Therefore, traditional MDP cannot be directly applied here. To solve this problem, we propose a 2-dimensional MDP (2DMDP) model, and a modified value iteration method to derive the network selection solutions for each user.

1) 2D-MDP Formulation: To construct the 2D-MDP model, we first need to define the actions of users and the network state transition probabilities. The action set of each user is choosing macrocell or femtocell network, denoted by $\mathcal{A}=$ $\{\mathbb{M}, \mathbb{F}\}$. Let $a_{s} \in A$ stand for a new user's action when arriving with network state $S$. In such a case, we can define the network state transition probabilities as follows:

$$
\begin{aligned}
& P_{n}\left\{S^{\prime} \mid S=\left(i_{m}, i_{f}\right)\right\} \\
& = \begin{cases}\lambda_{m}+\mathbf{1}_{\mathbb{M}}\left(a_{s}\right) \lambda, & \text { if } S^{\prime}=\left(i_{m}+1, i_{f}\right) \\
\lambda_{f}+\mathbf{1}_{\mathbb{F}}\left(a_{s}\right) \lambda, & \text { if } S^{\prime}=\left(i_{m}, i_{f}+1\right) \\
i_{m} \mu, & \text { if } S^{\prime}=\left(i_{m}-1, i_{f}\right) \\
i_{f} \mu, & \text { if } S^{\prime}=\left(i_{m}, i_{f}-1\right) \\
1-\lambda_{m}-\lambda_{f}-\lambda-\left(i_{m}+i_{f}\right) \mu, & \text { if } S^{\prime}=S=\left(i_{m}, i_{f}\right) \\
0, & \text { otherwise, }\end{cases}
\end{aligned}
$$

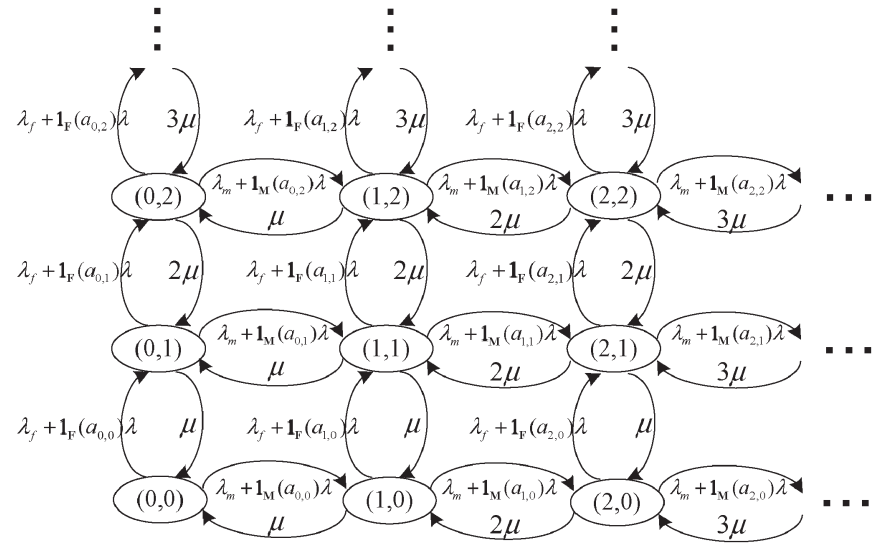

Fig. 4. State diagram of the 2-D Markov chain.

where $S^{\prime}$ is the next state of $S, \mu$ is the departure rate, and $\mathbf{1}_{\mathbb{M}}\left(a_{s}\right)$ and $\mathbf{1}_{\mathbb{F}}\left(a_{s}\right)$ are an indicator functions defined by

$$
\mathbf{1}_{\mathbb{M}}\left(a_{s}\right)\left\{\begin{array} { l l } 
{ 1 , } & { \text { if } a _ { s } = \mathbb { M } , } \\
{ 0 , } & { \text { if } a _ { s } = \mathbb { F } . }
\end{array} \quad \mathbf { 1 } _ { \mathbb { F } } ( a _ { s } ) \left\{\begin{array}{ll}
1, & \text { if } a_{s}=\mathbb{F}, \\
0, & \text { if } a_{s}=\mathbb{M} .
\end{array}\right.\right.
$$

The network parameters should be normalized to satisfy $\lambda_{m}+$ $\lambda_{f}+\lambda+\left(N_{m}+N_{f}\right) \mu<1$ in $P_{n}\left(S^{\prime}=S \mid S\right)$. Note that (25) are based on the assumption that the system time is discretized into small time slots, due to which the probability that more than one user arrive or leave simultaneously is very small and thus can be negligible [30]. With such an assumption, the state transition from one time slot to the next, with a non-negligible probability, can only be increasing 1 user, decreasing 1 user, or keeping unchanged. Moreover, when the time slot is sufficiently small, the probability that one user arrives or leaves within one slot is approximately equal to the arrival or departure rate, respectively. Fig. 4 illustrates the state transition diagram of the two-dimension Markov chain, where the staying probability $P_{n}\left(S^{\prime}=S \mid S\right)$ is not shown for conciseness.

Secondly, we need to define the expected utility functions of cognitive users. In general, each user will stay at the selected network for a period of time, during which the system state may change. Therefore, when making the network selection, the cognitive user should not only consider the immediate utility, but also take into account the future utilities. In the MDP model [31], Bellman equation is defined as a user's long-term expected payoff with the form as

$$
W\left(S_{0}\right)=\max _{\left\{a_{t}\right\}_{t=0}}\left\{U\left(S_{0}, a_{0}\right)+\sum_{t=1}^{\infty} \beta^{t} U\left(S_{t}, a_{t}\right)\right\},
$$

where the first term is the immediate utility of current state $S_{0}$, the second term is the expected utilities of the future states beginning from the initial state $S_{0}$, and $\beta^{t}$ is a discount factor series which ensures the summation is bounded. Bellman equation is usually written by a recursive form as follow

$$
W(S)=U(S)+\sum_{S^{\prime} \in \mathcal{X}} P\left(S^{\prime} \mid S, a_{s}\right) W\left(S^{\prime}\right),
$$

where $S^{\prime}$ represents the next state of $S$ and $P\left(S^{\prime} \mid S, a_{s}\right)$ is the state transition probability given the user's action at state $S, a_{s}$. According to the definition of Bellman equation, we can define 
a user's expected utility in macrocell network and femtocell network, $W_{m}(S)$ and $W_{f}(S)$, respectively as follows:

$$
\begin{gathered}
W_{m}\left(i_{m}, i_{f}\right)=U_{m}\left(i_{m}\right)+(1-\mu) \sum_{S^{\prime} \in \mathcal{X}} P_{m}\left(S^{\prime} \mid S\right) W_{m}\left(S^{\prime}\right), \\
W_{f}\left(i_{m}, i_{f}\right)=U_{f}\left(i_{f}\right)+(1-\mu) \sum_{S^{\prime} \in \mathcal{X}} P_{f}\left(S^{\prime} \mid S\right) W_{f}\left(S^{\prime}\right),
\end{gathered}
$$

where $(1-\mu)$ is the discount factor, which can be regarded as the probability that the user keeps staying at current network since $\mu$ is the departure probability; and $P_{m(f)}\left(S^{\prime} \mid S\right)$ in the second term is the state transition probability conditioned on that the user will still stay at current network in the next state $S^{\prime}$, which is different with the network state transition probability $P_{n}\left(S^{\prime} \mid S\right)$ in (25). Note that both $P_{m}\left(S^{\prime} \mid S\right)$ and $P_{f}\left(S^{\prime} \mid S\right)$ are closely related to the new arriving cognitive user's action and can be written as follows:

$$
\begin{aligned}
& P_{m}\left\{S^{\prime} \mid S=\left(i_{m}, i_{f}\right)\right\} \\
& = \begin{cases}\lambda_{m}+\mathbf{1}_{\mathbb{M}}\left(a_{s}\right) \lambda, & \text { if } S^{\prime}=\left(i_{m}+1, i_{f}\right) \\
\lambda_{f}+\mathbf{1}_{\mathbb{F}}\left(a_{s}\right) \lambda, & \text { if } S^{\prime}=\left(i_{m}, i_{f}+1\right) \\
\left(i_{m}-1\right) \mu, & \text { if } S^{\prime}=\left(i_{m}-1, i_{f}\right) \\
i_{f} \mu, & \text { if } S^{\prime}=\left(i_{m}, i_{f}-1\right) \\
1-\lambda_{m}-\lambda_{f}-\lambda-\left(i_{m}+i_{f}-1\right) \mu, & \text { if } S^{\prime}=S=\left(i_{m}, i_{f}\right) \\
0, & \text { otherwise, }\end{cases} \\
& P_{f} \begin{cases}\left.S^{\prime} \mid S=\left(i_{m}, i_{f}\right)\right\} & \text { if } S^{\prime}=\left(i_{m}+1, i_{f}\right)\end{cases} \\
& = \begin{cases}\lambda_{m}+\mathbf{1}_{\mathbb{M}}\left(a_{s}\right) \lambda, & \text { if } S^{\prime}=\left(i_{m}, i_{f}+1\right) \\
\lambda_{f}+\mathbf{1}_{\mathbb{F}}\left(a_{s}\right) \lambda, & \text { if } S^{\prime}=\left(i_{m}-1, i_{f}\right) \\
i_{m} \mu, & \text { if } S^{\prime}=\left(i_{m}, i_{f}-1\right) \\
\left(i_{f}-1\right) \mu, & \text { if } S^{\prime}=S=\left(i_{m}, i_{f}\right) \\
1-\lambda_{m}-\lambda_{f}-\lambda-\left(i_{m}+i_{f}-1\right) \mu, \\
0, & \text { otherwise, }\end{cases}
\end{aligned}
$$

where $a_{s}$ denotes the new arriving cognitive user's action, i.e., accessing whether macrocell network or femtocell network, and the terms $\left(i_{m}-1\right)$ in (31) and $\left(i_{m}-1\right)$ in (32) are because $i_{m}$ and $i_{f}$ already include the user who will not leave his/her current network at state $S^{\prime}$.

Unlike the traditional MDP problem with only one Bellman equation, there are two Bellman equations in our model as shown in (29) and (30), which we call as 2-dimensional MDP (2D-MDP) problem. The user's strategy profile $\pi=\left\{a_{s} \mid \forall S \in\right.$ $\mathcal{X}\}$ is a mapping from the state space to the action space, i.e., $\pi: \mathcal{X} \rightarrow \mathcal{A}$. Due to the selfish nature, each user will choose the best strategy to maximize his/her own expected utility. Suppose that one user arrives with system state $S=\left(i_{m}, i_{f}\right)$, his/her best strategy can be defined as

$$
a_{s}= \begin{cases}\mathbb{M}, & W_{m}\left(i_{m}+1, i_{f}\right) \geq W_{f}\left(i_{m}, i_{f}+1\right), \\ \mathbb{F}, & W_{m}\left(i_{m}, i_{f}+1\right)<W_{f}\left(i_{m}+1, i_{f}\right) .\end{cases}
$$

Since the strategy profile satisfying (29), (30), and (33), denoted by $\pi^{\star}$, maximizes every arriving user's utility, $\pi^{\star}$ is a Nash equilibrium.

2) Cognitive Users' Network Access Behavior: As discussed above, although the analysis of rational cognitive users' behavior can be modeled as an MDP problem, it is different from the traditional MDP problem that the user may not adjust action even if the network state changes. In traditional MDP problem, there is only one Bellman equation associated with each network state, and the best strategy is directly obtained by optimizing the Bellman equation. In our 2D-MDP problem, there are two Bellman equations associated with each network state as follows:

$$
\begin{aligned}
{\left[\begin{array}{l}
W_{m}(S) \\
W_{f}(S)
\end{array}\right]=} & {\left[\begin{array}{c}
U_{m}(S) \\
U_{f}(S)
\end{array}\right]+(1-\mu) } \\
& \cdot\left[\begin{array}{cc}
\mathbf{P}_{m}\left(S^{\prime} \mid S\right) & \mathbf{0} \\
\mathbf{0} & \mathbf{P}_{f}\left(S^{\prime} \mid S\right)
\end{array}\right]\left[\begin{array}{l}
\mathbf{W}_{m}^{T}\left(S^{\prime}\right) \\
\mathbf{W}_{f}^{T}\left(S^{\prime}\right)
\end{array}\right],
\end{aligned}
$$

where $\quad \mathbf{P}_{m(f)}\left(S^{\prime} \mid S\right)=\left[P_{m(f)}\left(S^{\prime} \mid S\right) \mid \forall S^{\prime} \in \mathcal{X}\right] \quad$ and $\mathbf{W}_{m(f)}\left(S^{\prime} \mid S\right)=\left[W_{m(f)}\left(S^{\prime} \mid S\right) \mid \forall S^{\prime} \in \mathcal{X}\right]$. Moreover, the best strategy profile $\pi^{\star}$ should satisfy (33) and (34) simultaneously. Therefore, the traditional dynamic programming method in [32] cannot be directly applied. To solve this problem, we design a modified value iteration algorithm.

Given an initial strategy profile $\pi$, the conditional state transition probability $\mathbf{P}_{m(f)}\left(S^{\prime} \mid S\right)$ can be calculated by (31) and (32), and thus the conditional expected utility $\mathbf{W}_{m(f)}(S)$ can be found by (34). Then, with $\mathbf{W}_{m(f)}(S)$, the strategy profile $\pi$ can be updated again using (33). Through such an iterative way, we can find the best strategy $\pi^{\star}$. In Algorithm 1, we summarize the proposed modified value iteration algorithm for the $2 \mathrm{D}$ MDP problem. The output of the algorithm is the best strategy for users, which is the network access behavior of the cognitive users defined at the beginning of Section IV. In the following, we will show that there exists a threshold structure in the best strategy profile $\pi^{\star}$.

Algorithm 1 Modified Value Iteration Algorithm for 2D-MDP Problem.

1: $\bullet$ Given tolerance $\Upsilon_{1}$ and $\Upsilon_{2}$, set $\epsilon_{1}$ and $\epsilon_{2}$.

2: - Initialize $\left\{W_{m(f)}^{(0)}(S)=0, \forall S \in \mathcal{X}\right\}$ and randomize $\pi=\left\{a_{s}, \forall S \in \mathcal{X}\right\}$.

3: while $\epsilon_{1}>\Upsilon_{1}$ or $\epsilon_{2}>\Upsilon_{2}$ do

4: for all $S \in \mathcal{X}$

5: $\quad$ - Calculate $\mathbf{P}_{m(f)}\left(S^{\prime} \mid S\right)$ using $\pi$ and (31), (32).

6: $\quad \bullet$ Update $\mathbf{W}_{m(f)}^{(n+1)}(S)$ using (34).

7: end for

8: for all $S \in \mathcal{X}$ do

9: $\quad$ Update $\pi^{\star}=\left\{a_{s}\right\}$ using (33).

10: end for

11: - Update the parameter $\epsilon_{1}$ by $\epsilon_{1}=\left\|\pi-\pi^{\star}\right\|_{2}$.

12: - Update the parameter $\epsilon_{2}$ by $\epsilon_{2}=\| \mathbf{W}_{m(f)}^{(n+1)}(S)-$ $\mathbf{W}_{m(f)}^{(n)}(S) \|_{2}$.

13: $\bullet$ Update the strategy file $\pi=\pi^{\star}$.

14: end while

15: • The best strategy profile is $\pi^{\star}$.

Lemma 1: For $i_{m} \geq 0$ and $i_{f} \geq 1$,

$$
\begin{aligned}
W_{m}\left(i_{m}, i_{f}\right) & \geq W_{m}\left(i_{m}+1, i_{f}-1\right) \\
W_{f}\left(i_{m}, i_{f}\right) & \leq W_{f}\left(i_{m}-1, i_{f}+1\right) .
\end{aligned}
$$

Proof: See Appendix A.

Lemma 1 shows that $W_{m}$ is non-decreasing and $W_{f}$ is non-increasing along the line of $i_{m}+i_{f}=m, \forall m \in$ $\left\{0,1, \ldots, N_{m}+N_{f}\right\}$. Based on Lemma 1, we will show the 
threshold structure in the best strategy profile $\pi^{\star}$ by following Theorem 2.

Theorem 2: The best strategy profile $\pi^{\star}=\left\{a_{s}\right\}$ derived from the modified value iteration algorithm has threshold structure as follows:

$$
\begin{aligned}
& \text { If } a_{s=\left(i_{m}, i_{f}\right)}=\mathbb{M}, \quad \text { then } a_{s=\left(i_{m}-i^{\prime}, i_{f}+i^{\prime}\right)}=\mathbb{M} . \\
& \text { If } a_{s=\left(i_{m}, i_{f}\right)}=\mathbb{F}, \quad \text { then } a_{s=\left(i_{m}+i^{\prime}, i_{f}-i^{\prime}\right)}=\mathbb{F} .
\end{aligned}
$$

Proof: See Appendix B.

Note that the best strategy profile $\pi^{\star}$ can be obtained off-line and the profile can be stored in a table in advance. We can see that the number of system states is $\left(N_{m}+1\right)\left(N_{f}+1\right)$, which means the corresponding strategy file has $\left(N_{m}+1\right)\left(N_{f}+1\right)$ strategies. With the proved threshold structure on each line $i_{m}+i_{f}=m, \forall m \in\left[0, N_{m}+N_{f}\right]$, we just need to store the threshold point on each line. In such a case, the storage of the strategy profile can be reduced from $\mathcal{O}\left(N^{2}\right)$ to $\mathcal{O}(2 N)$. Based on the analysis of users' network access behavior, we will further study the optimal pricing strategies for the operators in the following subsection.

\section{B. NE Price}

Given the user's network access behavior, i.e., the best strategy profile $\pi^{\star}=\left\{a_{s}\right\}$, the network state transition probability $P_{n}\left(S^{\prime} \mid S\right)$ can be obtained according to (25). With $\mathbf{P}_{n}\left(S^{\prime} \mid S\right)=\left\{P_{n}\left(S^{\prime} \mid S\right), \forall S^{\prime} \in \mathcal{X}\right\}$, we can then derive the stationary state probability distribution of Markov chain, $\sigma=$ $\{\sigma(S), \forall S \in \mathcal{X}\}$, by solving $\boldsymbol{\sigma} \mathbf{P}_{n}=\boldsymbol{\sigma}$. In network state $S=\left(i_{m}, i_{f}\right)$, the immediate utility of femtocell operator is $\left(p_{f}\left(i_{f}\right)-w\right) i_{f}$, while the immediate utility of macrocell operator is $p_{m}\left(i_{m}\right) i_{m}+w i_{f}$. In such a case, the expected utilities of macrocell and femtocell operators can be defined as

$$
\begin{aligned}
V_{m} & =\sum_{S=\left(i_{m}, i_{f}\right) \in \mathcal{X}} \sigma(S)\left(p_{m}\left(i_{m}\right) i_{m}+w i_{f}\right), \\
V_{f} & =\sum_{S=\left(i_{m}, i_{f}\right) \in \mathcal{X}} \sigma(S)\left(p_{f}\left(i_{f}\right)-w\right) i_{f} .
\end{aligned}
$$

On one hand, given the user's network access behavior $\pi^{\star}$, i.e., given the stationary state probability distribution $\sigma$, the NE price $\left(p_{m}^{N}\left(i_{m}\right), p_{f}^{N}\left(i_{f}\right)\right)$ can be obtained through maximizing (39) and (40). On the other hand, given the pricing strategy $\left(p_{m}\left(i_{m}\right), p_{f}\left(i_{f}\right)\right), \pi^{\star}$ can be calculated through Algorithm 1. Therefore, the final NE price $\left(p_{m}^{N}\left(i_{m}\right), p_{f}^{N}\left(i_{f}\right)\right)$ can be solved through iterative optimizations.

Algorithm 2 Iterative Gradient Descent Algorithm for Finding the NE Price.

1: • Given step size $\eta_{m(f)}$ and $\Delta_{m(f)}$, tolerance $\Upsilon_{1,2,3,4}$ and maximum iteration number $M A X$, set $\epsilon_{1,2,3,4}$.

2: • Initialize $l=0$, counter, $\mathbf{p}_{m}^{*(l)}$ and $\mathbf{p}_{f}^{*(l)}$ with random values that satisfy (23), (24).

3: while $\epsilon_{1}>\Upsilon_{1}$ and $\epsilon_{2}>\Upsilon_{2}$ and counter $<M A X$ do

4: $/ /^{* * * * * *}$ Fix $\mathbf{p}_{m}$, to find optimal $\mathbf{p}_{f}^{*} .^{* * * * * *} / /$

5: - Set $\mathbf{p}_{m}=\mathbf{p}_{m}^{*(l)}$, initialize $j=0$ and $\mathbf{p}_{f}^{(j)}$ with random value that satisfies (24).

6: $\quad$ while $\epsilon_{3}>\Upsilon_{3}$ do
7: $\quad$ Update femtocell operator's pricing strategy $\mathbf{p}_{f}^{(j+1)}$ using (41).

8: $\quad$ Update the parameter $\epsilon_{3}$ by $\epsilon_{3}=\left\|\mathbf{p}_{f}^{(j+1)}-\mathbf{p}_{f}^{(j)}\right\|_{2}$.

9: $\quad$ Update $\mathbf{p}_{f}^{*(l+1)}=\mathbf{p}_{f}^{(j+1)}$.

10: end while

11: $/ /{ }^{* * * * *}$ Fix $\mathbf{p}_{f}$, to find optimal $\mathbf{p}_{m}^{*} \cdot{ }^{* * * * *} / /$

12: $\bullet$ Set $\mathbf{p}_{f}=\mathbf{p}_{f}^{*(l)}$, initialize $k=0$ and $\mathbf{p}_{m}^{(k)}$ with random value that satisfies (23).

13: $\quad$ while $\epsilon_{4}>\Upsilon_{4}$ do

14: - Update macrocell operator's pricing strategy $\mathbf{p}_{m}^{(k+1)}$ using (42).

15: - Update the parameter $\epsilon_{4}$ by $\epsilon_{4}=\left\|\mathbf{p}_{m}^{(k+1)}-\mathbf{p}_{m}^{(k)}\right\|_{2}$.

16: $\quad$ Update $\mathbf{p}_{m}^{*(l+1)}=\mathbf{p}_{m}^{(k+1)}$.

17: end while

18: - Update the parameter $\epsilon_{1}$ by $\epsilon_{1}=\left\|\mathbf{p}_{m}^{*(l+1)}-\mathbf{p}_{m}^{*(l)}\right\|_{2}$.

19: - Update the parameter $\epsilon_{2}$ by $\epsilon_{2}=\left\|\mathbf{p}_{f}^{*(l+1)}-\mathbf{p}_{f}^{*(l)}\right\|_{2}$.

20: •Update $\left(\mathbf{p}_{m}^{N}, \mathbf{p}_{f}^{N}\right)=\left(\mathbf{p}_{m}^{*(l+1)}, \mathbf{p}_{f}^{*(l+1)}\right)$.

21: $\bullet$ Update counter $=$ counter +1 .

22: end while

23: • The NE price is $\left(\mathbf{p}_{m}^{N}, \mathbf{p}_{f}^{N}\right)$.

Let $\mathbf{p}_{m}=\left[p_{m}(1), \ldots, p_{m}\left(i_{m}\right), \ldots, p_{m}\left(N_{m}\right)\right]$ stand for the pricing strategy vector of macrocell operator, and $\mathbf{p}_{f}=$ $\left[p_{f}(1), \ldots, p_{f}\left(i_{f}\right), \ldots, p_{f}\left(N_{f}\right)\right]$ stand for that of femtocell operator. To find the NE price, we first fix $\mathbf{p}_{m}$ and calculate the optimal $\mathbf{p}_{f}^{*}$ which can maximize the femtocell operator's utility $V_{f}$, using gradient descent method by following update rule

$$
\begin{aligned}
p_{f}^{(j+1)}\left(i_{f}\right)=p_{f}^{(j)}\left(i_{f}\right)+\eta_{f} & \\
& \cdot \frac{V_{f}\left(\mathbf{p}_{m}, \mathbf{p}_{f}^{(j)}+\Delta_{f} \cdot \mathbf{e}\left(i_{f}\right)\right)-V_{f}\left(\mathbf{p}_{m}, \mathbf{p}_{f}^{(j)}\right)}{\Delta_{f}},
\end{aligned}
$$

where $\eta_{f}$ and $\Delta_{f}$ are step sizes, $\mathbf{e}\left(i_{f}\right)$ is a standard basis vector whose $i_{f}$-th coordinate is 1 and other coordinates are 0 , and the value of $V_{f}$ can be calculated by Algorithm 1 and (40). The second term of (41) is an approximation of the gradient of $V_{f}$ at $p_{f}^{j}\left(i_{f}\right)$. After obtaining the optimal $\mathbf{p}_{f}^{*}$, we then fix $\mathbf{p}_{f}=\mathbf{p}_{f}^{*}$ and calculate the optimal $\mathbf{p}_{m}^{*}$ which can maximize the macrocell operator's utility $V_{m}$, using gradient descent method by following update rule

$$
\begin{aligned}
& p_{m}^{(k+1)}\left(i_{m}\right)=p_{m}^{(k)}\left(i_{m}\right)+\eta_{m} \\
& \quad \frac{V_{m}\left(\mathbf{p}_{m}^{(k)}+\Delta_{m} \cdot \mathbf{e}\left(i_{m}\right), \mathbf{p}_{f}\right)-V_{m}\left(\mathbf{p}_{m}^{(k)}, \mathbf{p}_{f}\right)}{\Delta_{m}},
\end{aligned}
$$

where $\eta_{m}, \Delta_{m}$, and $\mathbf{e}\left(i_{m}\right)$ are similar to $\eta_{f}, \Delta_{f}$ and $\mathbf{e}\left(i_{f}\right)$ in (41), and the value of $V_{m}$ can be calculated by Algorithm 1 and (39). With optimal $\mathbf{p}_{m}^{*}$, we again fix $\mathbf{p}_{m}=\mathbf{p}_{m}^{*}$ and calculate $\mathbf{p}_{f}^{*}$ using (41). Through such an iterative optimization method, the convergence point $\left(\mathbf{p}_{m}^{*}, \mathbf{p}_{f}^{*}\right)$ satisfies that given macrocell's pricing strategy $\mathbf{p}_{m}^{*}$, femtocell operator's utility is maximized by price $\mathbf{p}_{f}^{*}$, while given femtocell's pricing strategy $\mathbf{p}_{f}^{*}$, macrocell operator's utility is maximized by price $\mathbf{p}_{m}^{*}$, i.e., $\left(\mathbf{p}_{m}^{*}, \mathbf{p}_{f}^{*}\right)$ is the NE price $\left(\mathbf{p}_{m}^{N}, \mathbf{p}_{f}^{N}\right)$ defined in Definition 1 . In Algorithm 2, we summarize the proposed iterative gradient descent algorithm to find the NE price. Note that although 
TABLE I

SIMULATION PARAMETERS

\begin{tabular}{|c|c|}
\hline Macrocell throughput & $R_{m}=2.5 \mathrm{Mbps}$ \\
\hline Femtocell throughput & $R_{f}=3.7 \mathrm{Mbps}$ \\
\hline Cognitive users' distribution & Hotelling distribution \\
\hline Cognitive users' arrival rates & $\lambda_{m}=\lambda_{f}=\lambda=0.02$ \\
\hline SNR & $\mathrm{SNR}_{m}=-13 \mathrm{~dB}, \mathrm{SNR}_{f}=-10 \mathrm{~dB}$ \\
\hline INR & $\mathrm{INR}_{m}=\mathrm{INR}_{f}=-14 \mathrm{~dB}$ \\
\hline Bandwidth & $B_{m}=B_{f}=8 M \mathrm{~Hz}$ \\
\hline Coefficient & $\theta=1000$ \\
\hline
\end{tabular}

it is difficult to theoretically analyze the convergence of this iterative optimization algorithm, we find that the proposed algorithm exhibits very fast convergence in simulations.

\section{Simulation Results}

In this section, we conduct simulations to further analyze the properties of the NE price, i.e., the influence of interference $\left(I_{m}, I_{f}\right)$ and spectrum leasing price $w$ on the NE price in the static pricing model, the influence of spectrum leasing price $w$ on the NE price in the dynamic pricing model, as well as the convergence performance of the proposed Algorithm 1 and 2. The parameters used in the simulation are listed in Table I.

\section{A. Static Pricing Model}

In this subsection, we discuss the simulation of static pricing model, where both network access prices $\left(p_{m}, p_{f}\right)$ are independent of the network state, i.e., the number of users. In the simulation, the throughput in macrocell and femtocell network are set as $R_{m}=2.5 \mathrm{Mbps}$ and $R_{f}=3.7 \mathrm{Mbps}$, respectively; the parameter $\theta$, which transfers the price into throughput loss, is set as $\theta=1000$.

We first illustrates the numerical results of NE price $\left(p_{m}^{N}, p_{f}^{N}\right)$ in Fig. 5, which shows the influence of interference in macrocell network $I_{m}$ and spectrum leasing prices $w$ on the NE price. In Fig. 5(a), we show the result with $w=2.5$, where we can see that when $I_{m} \leq 0.4 \mathrm{Mbps}, p_{m}^{N}$ keeps decreasing while $p_{f}^{N}$ keeps increasing. This is because with the increase of interference in macrocell network, macrocell operator has to decrease its price to attract more users, while femtocell operator can slightly increase its price to enhance utility. Such a phenomenon belongs to the second case of Theorem 1, i.e., "competition state". On the other hand, when $I_{m} \geq 0.4 \mathrm{Mbps}$, i.e., the interference in macrocell network is relatively severe, we can see that $p_{m}^{N}$ decreases with a faster speed, while $p_{f}^{N}$ remains steady. In this case, due to the high interference in macrocell network, only a small portion of users located near macrocell base station choose to access macrocell while most of users select femtocell network, which leads to the fact that femtocell's price $p_{f}^{N}$ only depends on its throughput $R_{f}$ and the spectrum leasing price $w$, as shown in the second case of Theorem 1. In Fig. 5(b), we show the results with $w=1.5$, where we can see that when $I_{m} \geq 0.8 \mathrm{Mbps}, p_{m}^{N}$ is equal to $w$, i.e., reaching the minimal value due to the severe interference in the macrocell network. For femtocell network, although $p_{f}^{N}$ keeps increasing, it is always smaller than that in "independent state". This shows that although $p_{m}^{N}$ is independent of $P_{f}^{N}$, it can suppress $P_{f}^{N}$ to be lower than $p_{f}^{I}$, which belongs to the "threatening state". Note that the gap between different states

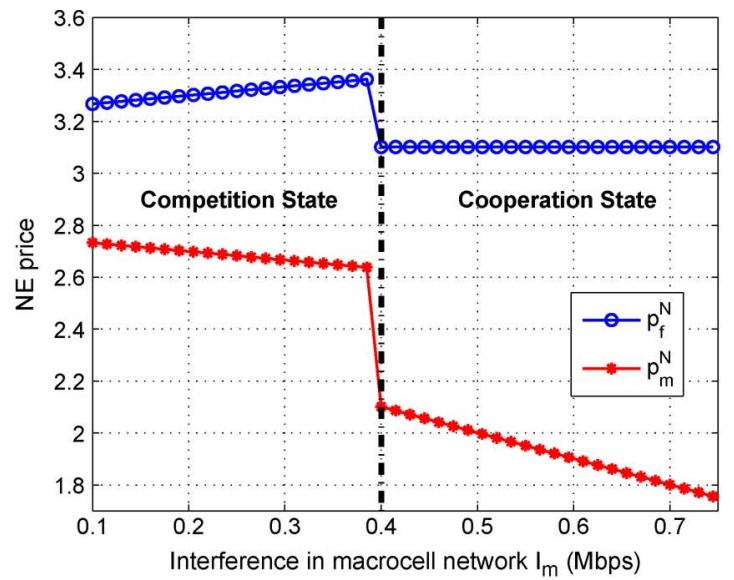

(a)

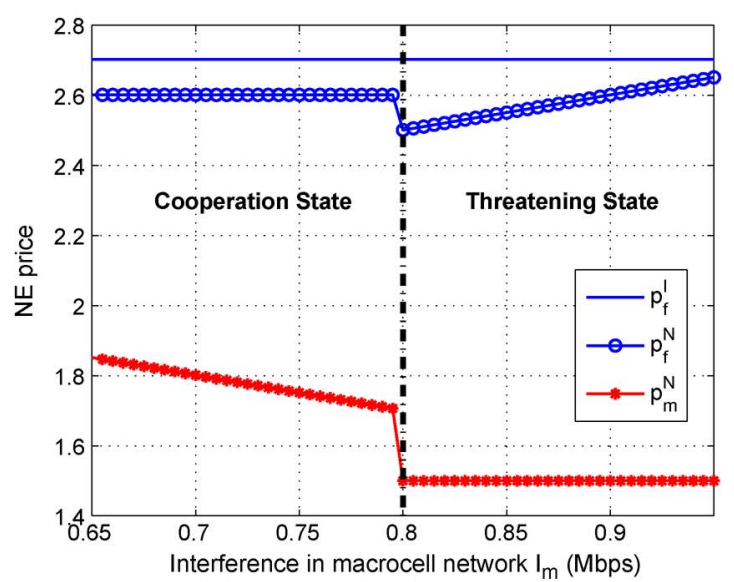

(b)

Fig. 5. NE price under different $I_{m}$ and $w$. (a) $w=2.5$. (b) $w=1.5$.

are due to state transition, which can be seen from the Nash pricing equations in Theorem 1.

We then illustrates the influence of interference in femtocell network $I_{f}$ and spectrum leasing prices $w$ on the NE price $\left(p_{m}^{N}, p_{f}^{N}\right)$ in Fig. 6. Similar to Fig. 5, there are also three different states under different settings of $I_{f}$ and $w$. The results with $w=0.9$ are shown in Fig. 6(a). We can see that when $I_{f} \leq 0.1 \mathrm{Mbps}$, although $p_{f}^{N}$ slowly decreases with the increase of $I_{f}$, most of users choose to access femtocell network due to the extremely low interference in femtocell network, which also leads to $p_{m}^{N}$ staying at the minimal value, i.e., the spectrum leasing price $w$. On the other hand, when $I_{f} \geq 0.1 \mathrm{Mbps}$, intuitively, $p_{f}^{N}$ should decrease with the increase of $I_{f}$. However, we can see from Fig. 6(a) that $p_{f}^{N}$ slowly increases with the increase of $I_{f}$. This is because, in this state, interference in femtocell network $I_{f}$ is relatively high but the spectrum leasing price $w$ is relatively low according to the condition of (17). In such a case, although the number of users accessing femtocell network $Q_{f}$ decreases with the increase of $I_{f}$, a higher $p_{f}^{N}$ can lead to a higher utility for femtocell operator due to the low spectrum leasing price $w$. In Fig. 6(b) where $w=1.5$, when $I_{f} \geq 0.7 \mathrm{Mbps}$, due to the high interference in femtocell network, there are only small portion of users accessing femtocell network. In this case, the network access price of both operators only rely on their own throughput, as shown in the second case of Theorem 1. 


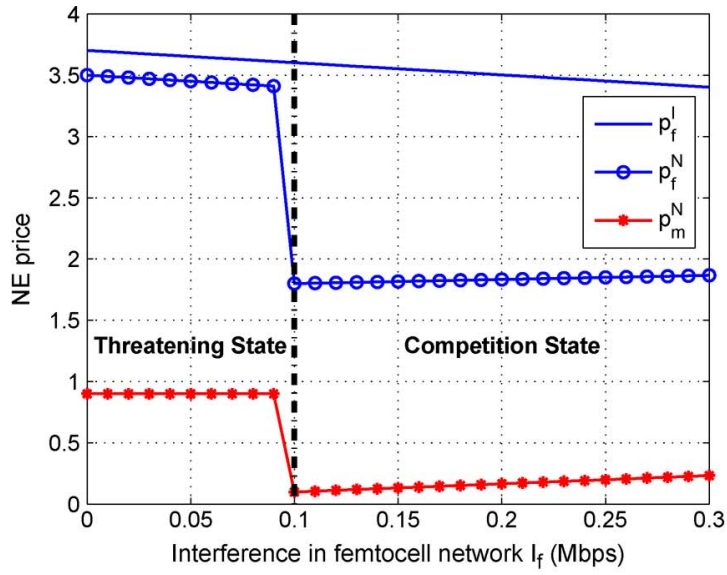

(a)

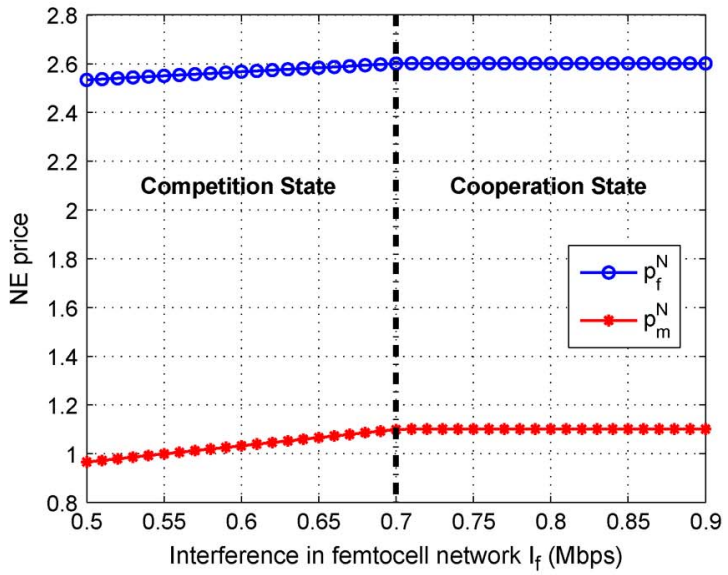

(b)

Fig. 6. NE price under different $I_{f}$ and $w$. (a) $w=0.9$. (b) $w=1.5$.

\section{B. Dynamic Pricing Model}

In this subsection, we discuss the simulation of dynamic pricing model, where both network access prices $\left(p_{m}, p_{f}\right)$ depend on the network state. In the simulation, the arrival rates of three kinds of users discussed at the beginning of Section IV are set as $\lambda_{m}=\lambda_{f}=\lambda=0.02$. The SNR in macrocell and femtocell networks are set as $\mathrm{SNR}_{m}=-13 \mathrm{~dB}$ and $\mathrm{SNR}_{f}=$ $-10 \mathrm{~dB}$; the INR in macrocell and femtocell networks are set as $\mathrm{INR}_{m}=\mathrm{INR}_{f}=-14 \mathrm{~dB}$ and the bandwidths are set as $B_{m}=B_{f}=8 \mathrm{MHz}$. The parameter $\theta$ is also set as $\theta=1000$.

We first verify the threshold structure of cognitive users' network access behavior shown in Theorem 2, i.e., the best strategy profile $\pi^{\star}$. Fig. 7 illustrates the strategy profile computed by Algorithm 1, where the maximum number of users in each network is set to be $N_{m}=N_{f}=10$, the network access price of two networks are set as $\mathbf{p}_{m}=\mathbb{1}_{10} * 1.5$ and $\mathbf{p}_{f}=$ $\mathbb{1}_{10} * 2.0$. The $x$-axis and $y$-axis denote the number of users in femtocell network and macrocell network, respectively, i.e., each coordinate $(x, y)$ is corresponding to one specific network state $\left(i_{f}, i_{m}\right)$. The $\mathbb{M}$ or $\mathbb{F}$ denotes the best strategy for users at this state, e.g., when $S=\left(i_{f}=3, i_{m}=5\right)$, the best strategy $a_{s}$ is $\mathbb{F}$ as marked by circle in the figure. We can see that the proposed algorithm converges in 20 iterations, which is denoted by the black line drawn on the boundary between $\mathbb{M}$ and $\mathbb{F}$. Moreover, the threshold lines of certain iterations (1,2, and 10) are also shown in the figure to illustrate the evolution of users'

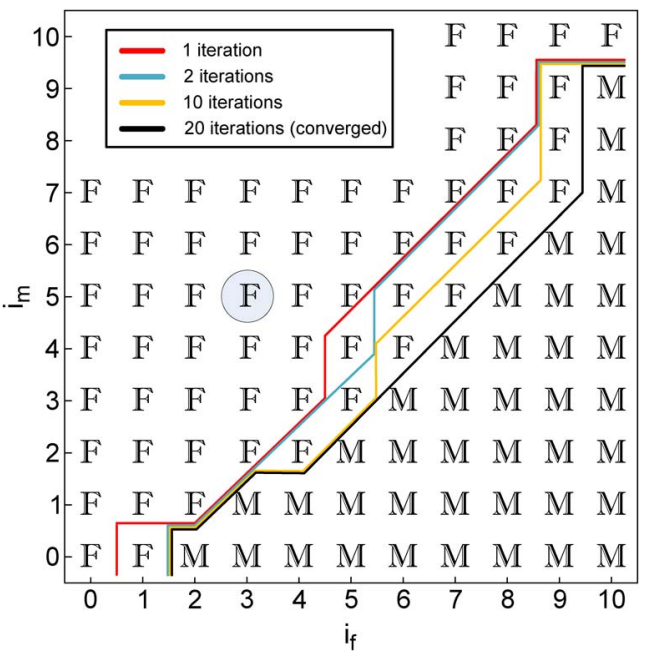

Fig. 7. Threshold structure of cognitive users' network access behavior.
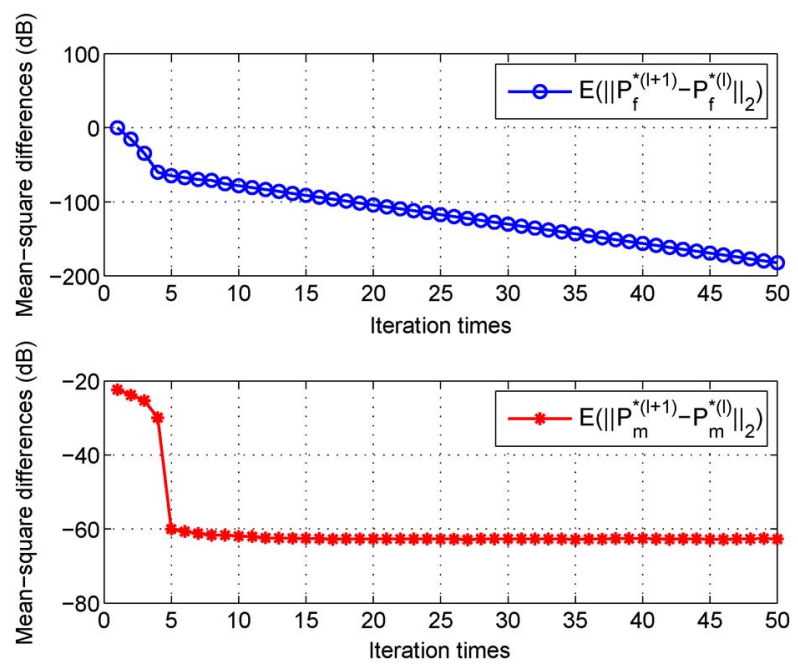

Fig. 8. Convergence performance of Algorithm 2.

behavior during the convergence process of Algorithm 1. It can be observed that the threshold structure always exists along the diagonal lines as analyzed in Section IV-A.2.

We then check the convergence performance of finding the NE price $\left(\mathbf{p}_{m}^{N}, \mathbf{p}_{f}^{N}\right)$ in Algorithm 2. Fig. 8 illustrates the convergence process of $\left(\mathbf{p}_{m}^{N}, \mathbf{p}_{f}^{N}\right)$ along with the simulation time, where the spectrum leasing price is set as $w=1$. In the figure, the $x$-axis denotes the iteration times and the $y$-axis denotes the mean-square differences of two adjacent iterations, i.e., $E\left(\left\|\mathbf{p}_{m(f)}^{*(l+1)}-\mathbf{p}_{m(f)}^{*(l)}\right\|_{2}\right)$. We can see that the proposed iterative gradient descent algorithm can converge to the NE price within 10 iterations, where the mean-square difference of $\mathbf{p}_{f}$ has already dropped to nearly $-100 \mathrm{~dB}$ and that of $\mathbf{p}_{m}$ has already dropped to $-60 \mathrm{~dB}$. Therefore, Algorithm 2 exhibits very fast convergence performance.

We further show the NE price $\left(\mathbf{p}_{m}^{N}, \mathbf{p}_{f}^{N}\right)$ computed by Algorithm 2 under different settings of spectrum leasing price $w$ in Fig. 9, where the maximum number of users in each network is set as $N_{m}=N_{f}=5$. For the NE price of dynamic pricing model, it is intuitive that the fewer number of users in the network, the higher throughput each user can obtain, and 

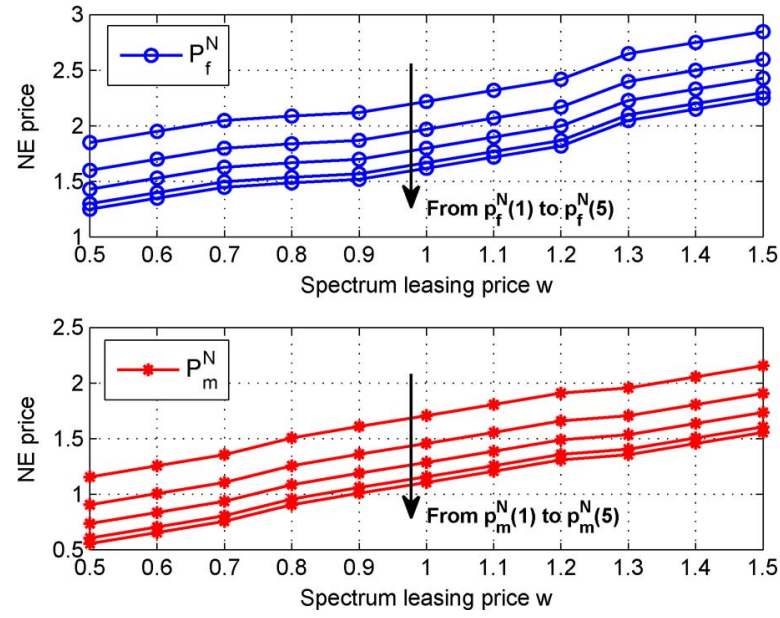

Fig. 9. NE price under different $w$.

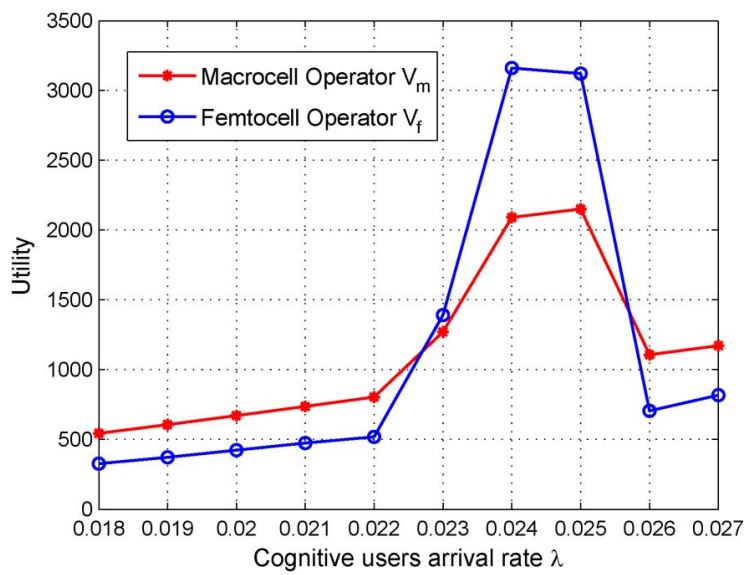

Fig. 10. Macrocell and femtocell operators' utilities.

the higher network access price should be set, i.e., $p_{m}^{N}\left(i_{m}\right)>$ $p_{m}^{N}\left(i_{m}+1\right)$ and $p_{f}^{N}\left(i_{f}\right)>p_{f}^{N}\left(i_{f}+1\right)$. From the figure, we can see the simulation results are consistent with the intuition that $p_{m(f)}^{N}(1)>p_{m(f)}^{N}(2)>p_{m(f)}^{N}(3)>p_{m(f)}^{N}(4)>p_{m(f)}^{N}(5)$. Moreover, we can also see that $\mathbf{p}_{f}^{N}>\mathbf{p}_{m}^{N}$. Such a phenomenon is because femtocell network can averagely provide higher data rate than macrocell network, and femtocell operator needs to pay the spectrum leasing price $w$ to macrocell operator. Fig. 9 also shows the influence of spectrum leasing price $w$ on the NE price $\left(\mathbf{p}_{m}^{N}, \mathbf{p}_{f}^{N}\right)$. We can see that both prices $\left(\mathbf{p}_{m}^{N}, \mathbf{p}_{f}^{N}\right)$ are larger than $w$ and they keep increase with the increasing of $w$. This phenomenon is similar to the "competition state" of static pricing model according to (17).

Moreover, we also show, in Fig. 10, the macrocell and femtocell operators' utilities, $V_{m}$ and $V_{f}$, under different settings of cognitive users' arrival rate $\lambda$. From the figure, we can see that with the increase of $\lambda, V_{m}$, and $V_{f}$ keep increasing when $\lambda<0.024$, since more and more users are joining macrocell or femtocell network. However, when $\lambda$ is larger than some threshold, $V_{m}$ and $V_{f}$ begin decreasing with the increase of $\lambda$. This phenomenon is because a high arrival rate can decrease the network access prices $p_{m}$ and $p_{f}$, which leads to the decrease of the utilities of both operators. Moreover, we can see that when users' arrival rate $\lambda<0.023$, the utility of macrocell operator is larger than that of femtocell operator, since accessing macrocell

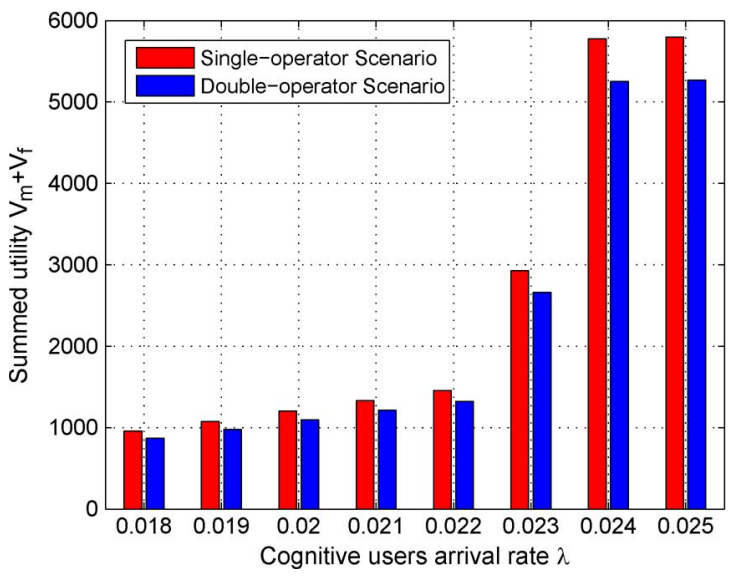

Fig. 11. Comparison between single-operator and double-operator scenarios.

operator with a lower price is preferred by cognitive users when arrival rate is relatively low. When $\lambda$ is in the middle, femtocell operator's utility becomes higher, which is because with the increasing number of users, accessing femtocell network can achieve more throughput. When $\lambda$ is relatively high, macrocell operator's utility becomes higher again, since both networks become crowded while macrocell operator has additional spectrum leasing revenue.

Finally, we conduct simulation to compare our work with the scenario where the macrocell and femtocell are operated by the same operator. In such a single-operator scenario, the operator can globally optimize the network access prices of both macrocell and femtocell by maximizing the sum utilities of them, which can be formulated as follows:

$$
\begin{aligned}
\max _{\left(p_{m}\left(i_{m}\right), p_{f}\left(i_{f}\right)\right)} & V_{m}+V_{f} \\
= & \sum_{S=\left(i_{m}, i_{f}\right) \in \mathcal{X}} \sigma(S)\left(p_{m}\left(i_{m}\right) i_{m}+p_{f}\left(i_{f}\right)\right) .
\end{aligned}
$$

For the cognitive users, they still access the network that can offer higher utility and make the network access decision using Algorithm 1 . The optimal price $\left(p_{m}^{*}\left(i_{m}\right), p_{f}^{*}\left(i_{f}\right)\right)$ of the singleoperator scenario can also be found through the iterative way, i.e., first fixing the price $\left(p_{m}\left(i_{m}\right), p_{f}\left(i_{f}\right)\right)$ to obtain the stationary state probability distribution $\sigma(S)$ using Algorithm 1, then calculating the optimal $\left(p_{m}^{*}\left(i_{m}\right), p_{f}^{*}\left(i_{f}\right)\right)$ by solving (43) based on the obtained $\sigma(S)$, and so forth. Fig. 11 shows the summed utility comparison results between our work (denoted by double-operator scenario) and the single-operator scenario, where the $x$-axis is the cognitive users' arrival rate $\lambda$. We can see that the single-operator scenario performs better than the double-operator scenario. This is because in the single-operator scenario, the operator can globally maximize the summed utility of both macrocell and femtocell, i.e., the objective is just to maximize the comparison metric $V_{m}+V_{f}$, while in the doubleoperator scenario discussed in this paper, each operator only maximizes its own utility and the Nash equilibrium is achieved. However, the single-operator scenario can be only applied to the monopoly based market which is rarely seen in the real-world market, while the double-operator scenario can be well applied in the competition-based market and provide an equilibrium point for the market, which is more common in the current practical scenario. 


\section{CONCLUSION}

In this paper, we analyzed the NE price of macrocell and femtocell operators through studying the two-tier pricing model. In static pricing model, we derived the closed-form expressions for the pricing and demand functions, as well as the NE price. In dynamic pricing model, we modeled the cognitive users' behaviors as a 2D-MDP model and designed a modified value iteration algorithm to derive the best network access strategy for users. Based on the analysis of users' behavior, we then designed an iterative gradient descent algorithm to find the NE price of both operators. According to the simulation results, we further analyzed the influence of spectrum leasing price on the NE price of both models. In this paper, we only discussed the scenario when the femtocell and macrocell are not operated by the same service provider. In the future, we will study the hybrid model where femtocell and macrocell may be operated by same service provider or not.

\section{APPENDIX}

\section{A. Proof of Lemma 1}

We use induction method to prove that (35) and (36) hold for all $n \geq 0$. First, since $W_{m}^{(0)}\left(i_{m}, i_{f}\right)$ and $W_{f}^{(0)}\left(i_{m}, i_{f}\right)$ are initialized by zeros in Algorithm 1, (35) and (36) hold for $n=$ 0 . Second, we assume that (35) and (36) hold for some $n>0$, and check whether (35) and (36) hold for $(n+1)$. For notation simplicity, we use $S_{1}=\left(i_{m}, i_{f}\right)$ and $S_{2}=\left(i_{m}+1, i_{f}-1\right)$. There are three cases for action $a_{s_{1}}^{(n)}$ and action $a_{s_{2}}^{(n)}$ :

- Case 1: $W_{f}^{(n)}\left(S_{1}\right) \leq W_{f}^{(n)}\left(S_{2}\right) \leq W_{m}^{(n)}\left(S_{2}\right) \leq W_{m}^{(n)}\left(S_{1}\right)$, we have $a_{s_{1}}^{(n)}=a_{s_{2}}^{(n)}=\mathbb{M}$;

- Case 2: $W_{m}^{(n)}\left(S_{2}\right) \leq W_{m}^{(n)}\left(S_{1}\right) \leq W_{f}^{(n)}\left(S_{1}\right) \leq W_{f}^{(n)}\left(S_{2}\right)$, we have $a_{s_{1}}^{(n)}=a_{s_{2}}^{(n)}=\mathbb{F}$;

- Case 3: $W_{m}^{(n)}\left(S_{1}\right) \geq W_{f}^{(n)}\left(S_{1}\right)$ and $W_{m}^{(n)}\left(S_{2}\right) \leq W_{f}^{(n)}\left(S_{2}\right)$ we have $a_{s_{1}}^{(n)}=\mathbb{M}$ and $a_{s_{2}}^{(n)}=\mathbb{F}$.

For Case 1 , we have the difference of $W_{m}\left(i_{m}, i_{f}\right)$ and $W_{m}\left(i_{m}+1, i_{f}-1\right)$ as follows:

$$
\begin{aligned}
& W_{m}^{(n+1)}\left(S_{1}\right)-W_{m}^{(n+1)}\left(S_{2}\right) \\
& =\left(U_{m}\left(i_{m}\right)-U_{m}\left(i_{m}+1\right)\right)+(1-\mu) \\
& \times\left[\lambda\left(W_{m}^{(n)}\left(i_{m}+1, i_{f}\right)-W_{m}^{(n)}\left(i_{m}+2, i_{f}-1\right)\right)+\lambda_{2}\right. \\
& \quad \times\left(W_{m}^{(n)}\left(i_{m}, i_{f}+1\right)-W_{m}^{(n)}\left(i_{m}+1, i_{f}\right)\right) \\
& \quad+\lambda_{1}\left(W_{m}^{(n)}\left(i_{m}+1, i_{f}\right)-W_{m}^{(n)}\left(i_{m}+2, i_{f}-1\right)\right) \\
& \quad+\mu\left(i_{f}-1\right)\left(W_{m}^{(n)}\left(i_{m}, i_{f}-1\right)-W_{m}^{(n)}\left(i_{m}+1, i_{f}-2\right)\right) \\
& \quad+\mu\left(i_{m}-2\right)\left(W_{m}^{(n)}\left(i_{m}-1, i_{f}\right)-W_{m}^{(n)}\left(i_{m}, i_{f}-1\right)\right) \\
& \quad+\left(1-\lambda_{1}-\lambda_{2}-\lambda-\left(i_{m}+i_{f}-1\right) \mu\right) \\
& \left.\quad \cdot\left(W_{m}^{(n)}\left(i_{m}, i_{f}\right)-W_{m}^{(n)}\left(i_{m}+1, i_{f}-1\right)\right)\right] .
\end{aligned}
$$

Since $U_{m}\left(i_{m}\right)$ is a decreasing function in terms of $i_{m}$ defined in (21), with the hypothesis that $W_{m}^{(n)}\left(S_{1}\right)-W_{f}^{(n)}\left(S_{2}\right) \geq 0$, we can see that $W_{m}^{(n+1)}\left(S_{1}\right)-W_{f}^{(n+1)}\left(S_{2}\right) \geq 0$ holds according to (44). For Cases 2 and 3, same conclusions can be obtained by analyzing the difference of $W_{m}^{(n+1)}\left(S_{1}\right)$ and $W_{m}^{(n+1)}\left(S_{2}\right)$. Thus, we conclude that $W_{m}\left(S_{1}\right) \geq W_{m}\left(S_{2}\right)$. Similarly, $W_{f}\left(S_{1}\right) \leq W_{f}\left(S_{2}\right)$ can be proved by induction. Here, due to page limitation, we skip the detailed proof.

\section{B. Proof of Theorem 2}

According to Lemma 1, we can have

$$
\begin{aligned}
& W_{m}\left(i_{m}+1, i_{f}\right)-W_{f}\left(i_{m}, i_{f}+1\right) \\
& \quad \geq W_{m}\left(i_{m}+2, i_{f}-1\right)-W_{f}\left(i_{m}+1, i_{f}\right),
\end{aligned}
$$

which shows that the difference of $W_{m}$ and $W_{f}$ is nondecreasing along the line $i_{m}+i_{f}=m, \forall m \in\{0,1, \ldots$, $\left.N_{m}+N_{f}\right\}$. In such a case, on one hand, if $W_{m}\left(i_{m}+\right.$ $\left.1, i_{f}\right) \leq W_{f}\left(i_{m}, i_{f}+1\right)$, i.e., $a_{s=\left(i_{m}, i_{f}\right)}=\mathbb{F}$, then for any $i^{\prime}>$ $0, W_{m}\left(i_{m}+i^{\prime}+1, i_{f}-i^{\prime}\right) \leq W_{f}\left(i_{m}+i^{\prime}, i_{f}-i^{\prime}+1\right)$, i.e., $a_{s=\left(i_{m}+i^{\prime}, i_{f}-i^{\prime}\right)}=\mathbb{F}$. On the other hand, if $W_{m}\left(i_{m}+1, i_{f}\right) \geq$ $W_{f}\left(i_{m}, i_{f}+1\right)$, i.e., $a_{s=\left(i_{m}, i_{f}\right)}=\mathbb{M}$, then for any $i^{\prime}>$ $0, W_{m}\left(i_{m}-i^{\prime}+1, i_{f}+i^{\prime}\right) \geq W_{f}\left(i_{m}-i^{\prime}, i_{f}+i^{\prime}+1\right)$ which means $a_{s=\left(i_{m}-i^{\prime}, i_{f}+i^{\prime}\right)}=\mathbb{M}$. Therefore, we can conclude that if $a_{s=\left(i_{m}, i_{f}\right)}=\mathbb{M}$, then the upper left of line $i_{m}+i_{f}=m$ will be all $\mathbb{M}$, and if $a_{s=\left(i_{m}, i_{f}\right)}=\mathbb{F}$, then the lower right of line $i_{m}+i_{f}=m$ will be all $\mathbb{F}$. Thus, there exists some threshold on the line of $i_{m}+i_{f}=m$.

\section{REFERENCES}

[1] S. Al-Rubaye, A. Al-Dulaimi, and J. Cosmas, "Cognitive femtocell," IEEE Veh. Technol. Mag., vol. 6, no. 3, pp. 44-51, Mar. 2011.

[2] K. J. R. Liu and B. Wang, Cognitive Radio Networking and Security: A Game Theoretical View. Cambridge, U.K.: Cambridge Univ. Press, 2010.

[3] G. Gur, S. Bayhan, and F. Alagoz, "Cognitive femtocell networks: An overlay architecture for localized dynamic spectrum access," IEEE Wireless Commun., vol. 17, no. 4, pp. 62-70, Aug. 2010.

[4] C. Jiang, Y. Chen, K. J. R. Liu, and Y. Ren, "Renewal-theoretical dynamic spectrum access in cognitive radio networks with unknown primary behavior," IEEE J. Sel. Areas Commun., vol. 31, no. 3, pp. 406-416, Mar. 2013.

[5] J. Xiang, Y. Zhang, T. Skeie, and L. Xie, "Downlink spectrum sharing for cognitive radio femtocell networks," IEEE Syst. J., vol. 4, no. 4, pp. 524-534, Dec. 2010.

[6] X. Chu, Y. Wu, D. Lopez-Perez, and X. Tao, "On providing downlink services in collocated spectrum-sharing macro and femto networks," IEEE Trans. Wirelesss Commun., vol. 10, no. 12, pp. 4306-4315, Dec. 2011.

[7] S. Cheng, W. Ao, F. Tseng, and K. Chen, "Design and analysis of downlink spectrum sharing in two-tier cognitive femto networks," IEEE Trans. Veh. Technol., vol. 61, no. 5, pp. 2194-2207, Jun. 2012.

[8] C.-W. Chang, "An interference-avoidance code assignment strategy for the hierarchical two-dimensional-spread MC-DS-CDMA system: A prototype of cognitive radio femtocell system," IEEE Trans. Veh. Technol., vol. 61, no. 1, pp. 166-184, Jan. 2012.

[9] X. Y. Wang, P.-H. Ho, and K.-C. Chen, "Interference analysis and mitigation for cognitive-empowered femtocells through stochastic dual control," IEEE Trans. Wirelesss Commun., vol. 11, no. 6, pp. 2065-2075, Jun. 2012.

[10] J. W. Huang and V. Krishnamurthy, "Cognitive base stations in LTE/3GPP femtocells: A correlated equilibrium game-theoretic approach," IEEE Trans. Commun., vol. 59, no. 12, pp. 3485-3493, Dec. 2011.

[11] R. Xie, F. R. Yu, H. Ji, and Y. Li, "Energy-efficient resource allocation for heterogeneous cognitive radio networks with femtocells," IEEE Trans. Wirelesss Commun., vol. 11, no. 11, pp. 3910-3920, Nov. 2012.

[12] R. Urgaonkar and M. J. Neely, "Opportunistic cooperation in cognitive femtocell networks," IEEE J. Sel. Areas Commun., vol. 30, no. 3, pp. 607616, Apr. 2012.

[13] D. Hu and S. Mao, "On medium grain scalable video streaming over femtocell cognitive radio networks," IEEE J. Sel. Areas Commun., vol. 30, no. 3, pp. 641-651, Apr. 2012. 
[14] S.-Y. Yun, Y. Yi, D.-H. Cho, and J. Mo, "The economic effects of sharing femtocells," IEEE J. Sel. Areas Commun., vol. 30, no. 3, pp. 595-606, Apr. 2012.

[15] X. Kang, R. Zhang, and M. Motani, "Price-based resource allocation for spectrum-sharing femtocell networks: A Stackelberg game approach," IEEE J. Sel. Areas Commun., vol. 30, no. 3, pp. 538-549, Apr. 2012.

[16] S. Ren, J. Park, and M. van der Schaar, "Entry and spectrum sharing scheme selection in femtocell communications markets," IEEE/ACM Trans. Netw., vol. 21, no. 1, pp. 218-232, Feb. 2013.

[17] L. Duan, J. Huang, and B. Shou, "Economics of femtocell service provision," IEEE Trans. Mobile Comput., vol. 12, no. 11, pp. 2261-2273, Nov. 2013

[18] W. Worldlive, Report: China Unicom, China Telecom awarded FDDLTE spectrum. [Online]. Available: http://www.mobileworldlive.com/ report-china-unicom-china-mobile-awarded-fdd-lte-spectrum

[19] F. Pantisano, M. Bennis, W. Saad, and M. Debbah, "Spectrum leasing as an incentive towards uplink macrocell and femtocell cooperation," IEEE J. Sel. Areas Commun., vol. 30, no. 3, pp. 617-630, Apr. 2012.

[20] Y. Yi, J. Zhang, Q. Zhang, and T. Jiang, "Spectrum leasing to femto service provider with hybrid access," in Proc. IEEE INFOCOM, 2012, pp. $1215-1223$.

[21] S. K. Jayaweera, G. Vazquez-Vilar, and C. Mosquera, "Dynamic spectrum leasing: A new paradigm for spectrum sharing in cognitive radio networks," IEEE Trans. Veh. Technol., vol. 59, no. 5, pp. 2328-2339, Jun. 2010.

[22] G. El-Howayek and S. K. Jayaweera, "Distributed dynamic spectrum leasing (D-DSL) for spectrum sharing over multiple primary channels," IEEE Trans. Wireless Commun., vol. 10, no. 1, pp. 55-60, Jan. 2011.

[23] S. K. Jayaweera, M. Bkassiny, and K. A. Avery, "Asymmetric cooperative communications based spectrum leasing as an incentive towards uplink macrocell and femtocell cooperation," IEEE Trans. Wireless Commun., vol. 10, no. 8, pp. 2716-2724, Aug. 2011.

[24] C.-Y. Wang and H.-Y. Wei, "Profit maximization in femtocell service with contract design," IEEE Trans. Wireless Commun., vol. 12, no. 5, pp. 1978-1988, May 2013.

[25] C. Jiang, Y. Chen, Y. Gao, and K. J. R. Liu, "Joint spectrum sensing and access evolutionary game in cognitive radio networks," IEEE Trans. Wireless Commun., vol. 12, no. 5, pp. 2470-2483, May 2013

[26] C. Jiang, Y. Chen, and K. J. R. Liu, "Multi-channel sensing and access game: Bayesian social learning with negative network externality," IEEE Trans. Wireless Commun., vol. 13, no. 4, pp. 2176-2188, Apr. 2014.

[27] Y. Yang, Y. Chen, C. Jiang, C. Wang, and K. J. R. Liu, "Wireless access network selection game with negative network externality," IEEE Trans. Wireless Commun., vol. 12, no. 10, pp. 5048-5060, Oct. 2013.

[28] H. Hotelling, "Stability in competition," Econ. J., vol. 39, no. 153, pp. 41-57, Mar. 1929.

[29] V. Chandrasekhar, J. G. Andrews, and A. Gatherer, "Femtocell networks: A survey," IEEE Commun. Mag., vol. 46, no. 9, pp. 59-67, Sep. 2008.

[30] C. Jiang, Y. Chen, Y. Yang, C. Wang, and K. J. R. Liu, "Dynamic Chinese restaurant game: Theory and application to cognitive radio networks," IEEE Trans. Wireless Commun., vol. 13, no. 4, pp. 1960-1973, Apr. 2014.

[31] M. L. Puterman, Markov Decision Processes: Discrete Stochastic Dynamic Programming. Hoboken, NJ, USA: Wiley, 1994.

[32] D. Bertsekas, Dynamic Programming and Optimal Control, 3rd ed. Belmont, MA, USA: Athena Scientific, 2007.

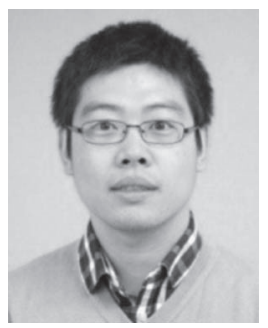

Chunxiao Jiang (S'09-M'13) received the B.S. degree in information engineering from Beijing University of Aeronautics and Astronautics (currently Beihang University), Beijing, China, in 2008 and the Ph.D. degree from Tsinghua University (THU), Beijing, in 2013, both with the highest honors. During 2011-2013, he visited the Signals and Information Group, Department of Electrical and Computer Engineering, University of Maryland, College Park, MD, USA, with Prof. K. J. Ray Liu. Dr. Jiang is currently a Postdoctor with the Department of Electrical Engineering, THU, with Prof. Y. Ren. His research interests include the applications of game theory and queuing theory in wireless communication and networking and social networks.

Dr. Jiang was the recipient of the Best Paper Award at the IEEE GLOBECOM in 2013 and the Beijing Distinguished Graduated Student Award, Chinese National Fellowship, and Tsinghua Outstanding Distinguished Doctoral Dissertation in 2013.

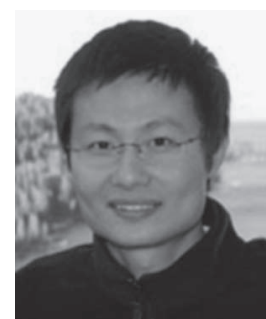

Yan Chen (S'06-M'11) received the B.S. degree from the University of Science and Technology of China, Hefei, China, in 2004, the M.Phil. degree from Hong Kong University of Science and Technology, Kowloon, Hong Kong, in 2007, and the Ph.D. degree from the University of Maryland, College Park, MD, USA, in 2011. His current research interests are in data science, network science, game theory, social learning and networking, as well as signal processing and wireless communications.

Dr. Chen was the recipient of multiple honors and awards, including the Best Paper Award at the IEEE GLOBECOM in 2013; Future Faculty Fellowship and Distinguished Dissertation Fellowship Honorable Mention from the Department of Electrical and Computer Engineering in 2010 and 2011, respectively; Finalist of Deans Doctoral Research Award from A. James Clark School of Engineering, University of Maryland in 2011; and Chinese Government Award for Outstanding Students Abroad in 2011.

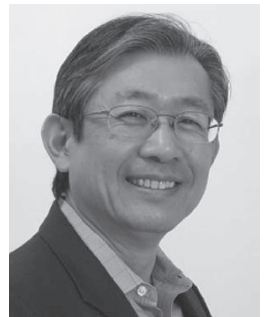

K. J. Ray Liu (F'03) was named a Distinguished Scholar-Teacher of the University of Maryland, College Park, MD, USA, in 2007, where he is Christine Kim Eminent Professor of Information Technology. He leads the Maryland Signals and Information Group, conducting research encompassing broad areas of signal processing and communications with recent focus on cooperative and cognitive communications, social learning and network science, information forensics and security, and green information and communications technology.

Dr. Liu was a Distinguished Lecturer and a recipient of the IEEE Signal Processing Society 2009 Technical Achievement Award and various best paper awards. He also received various teaching and research recognitions from the University of Maryland, including the university-level Invention of the Year Award, as well as Poole and Kent Senior Faculty Teaching Award, Outstanding Faculty Research Award, and Outstanding Faculty Service Award, all from A. James Clark School of Engineering. An ISI Highly Cited Author, Dr. Liu is a Fellow of AAAS.

Dr. Liu is a Past President of the IEEE Signal Processing Society, where he has served as President, Vice President-Publications, and Board of Governor He was the Editor-in-Chief of the IEEE Signal Processing Magazine and the founding Editor-in-Chief of EURASIP Journal on Advances in Signal Processing.

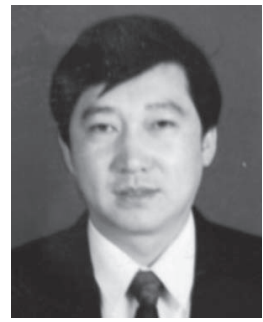

Yong Ren (M'11) received the B.S., M.S., and Ph.D. degrees in electronic engineering from Harbin Institute of Technology, Harbin, China, in 1984, 1987, and 1994, respectively. He was a Postdoctor with the Department of Electronics Engineering, Tsinghua University, Beijing, China, from 1995 to 1997. Now, he is a Professor with the Department of Electronics Engineering and the Director of the Complexity Engineered Systems Laboratory, Tsinghua University. He holds 12 patents and has authored or coauthored more than 100 technical papers in the behavior of computer networks, P2P networks, and cognitive networks. His current research interests include complex systems theory and its applications to the optimization and information sharing of the Internet, space-based information networks, Internet of Things and ubiquitous networks, cognitive networks, and cyber-physical systems. 